\title{
Nonrelativistic superparticle in a curved background
}

\author{
Eric Bergshoeff, ${ }^{1, *}$ Joaquim Gomis, ${ }^{2, \dagger}$ Marija Kovačević, ${ }^{1, \$}$ Lorena Parra, ${ }^{1,3, \S}$ Jan Rosseel, ${ }^{4, \|}$ and Thomas Zojer ${ }^{1, \|}$ \\ ${ }^{1}$ Van Swinderen Institute for Particle Physics and Gravity, University of Groningen, Nijenborgh 4, \\ 9747 AG Groningen, Netherlands \\ ${ }^{2}$ Departament d'Estructura i Constituents de la Matèria and Institut de Ciències del Cosmos, \\ Universitat de Barcelona, Diagonal 645, 08028 Barcelona, Spain \\ ${ }^{3}$ Instituto de Ciencias Nucleares, Universidad Nacional Autónoma de México, Apartado Postal 70-543, \\ 04510 México, Distrito Federal, México \\ ${ }^{4}$ Institute for Theoretical Physics, Vienna University of Technology, Wiedner Hauptstrasse 8-10/136, \\ A-1040 Vienna, Austria
}

(Received 10 July 2014; published 4 September 2014)

\begin{abstract}
Using a component formulation, we construct the supersymmetric action for a superparticle in a threedimensional Newton-Cartan supergravity background and clarify its symmetries. Our construction proceeds by first constructing the superparticle in a flat background. Next, by boosting up the background symmetries, we replace in a first step the flat background by a Galilean supergravity background. In a second step the Galilean supergravity background is replaced by a Newton-Cartan supergravity background. We extend our results by adding a supersymmetric cosmological constant and compare the nonrelativistic superparticle with the relativistic $\kappa$-symmetric three-dimensional superparticle.
\end{abstract}

\section{INTRODUCTION}

Over the past few years there has been an increased interest in extending the holographic principle to nonrelativistic theories. The main motivation is to widen the applications of the conjectured AdS/CFT correspondence and to test if it also holds away from its original relativistic setting. There are reasons to expect that nonrelativistic holography has applications in effective descriptions of strongly correlated condensed matter systems [1-5]; for a review, see e.g. Ref. [6]. Usually, one only considers a nonrelativistic setting at the boundary, but one might also consider nonrelativistic models both in the bulk and at the boundary [7]. In the context of the AdS/CFT correspondence nonrelativistic theories also occur as boundary geometries. For example, the authors of Refs. $[8,9]$ have found that gravity theories supporting Lifshitz geometries lead to a boundary geometry that is characterized by a socalled torsional Newton-Cartan geometry. ${ }^{1}$ More recently, nonrelativistic gravity and Newton-Cartan structures, see e.g. Refs. $[10,11]$, have also been used as a geometric way of realizing nonrelativistic symmetries of effective field theories [12]. Originally, before all these developments took place, nonrelativistic superstring theories and superbranes were studied as special points in the parameter space

\footnotetext{
e.a.bergshoeff@rug.nl

joaquim.gomis@gmail.com

*m.kovacevic@rug.nl

\$1.parra.rodriguez@rug.nl

rosseelj@gmail.com

t.zojer@rug.nl

${ }^{1}$ The torsion occurs due to the fact that the boundary geometry is invariant under an anisotropic scaling symmetry.
}

of $M$-theory with nonrelativistic symmetries [13,14]. Nonrelativistic strings were also thought as a possible soluble sector within string theory or $M$-theory $[15,16]$. This latter expectation was based on a similar experience with the pp-wave/Berenstein-Maldacena-Nastase limit [17].

The goal of this paper is to make a first step in improving our knowledge on nonrelativistic gravity and particle/ string/brane theories that underly a holography with a nonrelativistic setting at both the boundary and the bulk. In this work we will consider supersymmetric theories, and we will restrict to particles, or more precisely superparticles $[18,19]$, only.

Nonrelativistic $\mathcal{N}=2$ massive superparticles in ten dimensions in a flat background have already been studied in Ref. [15]. In this paper we wish to extend this analysis and consider superparticles in a curved nonrelativistic supergravity background. It should be stressed that, independent of the relation with nonrelativistic holography, there is not much literature on nonrelativistic supersymmetry; however, see e.g. Refs. [20-26]. This in itself provides ample reason to investigate this topic.

Part of this paper consists of a review of known results on nonrelativistic superparticles in a flat background. To the best of our knowledge, all nonrelativistic superparticle actions with background fields, with or without a cosmological constant, are new.

In the construction of massive (super)particle actions an important role is played by symmetries, both global and local ones. In particular, it is well known that relativistic massive superparticles have an infinitely reducible gauge symmetry, called $\kappa$-symmetry [27,28], that eliminates half of the fermions. In the nonrelativistic setting this 
TABLE I. This table gives an overview of the different bosonic backgrounds used in this paper (first column). The second and third columns indicate the names we use for a particle moving in such backgrounds plus the symmetries of the corresponding particle action in the case of a zero cosmological constant $(\Lambda=0)$. The last two columns give the same information for the case of a nonzero cosmological constant $(\Lambda \neq 0)$. We have denoted the accelerationextended Galilei symmetries with "a.e. Galilei" while g.c.t. stands for general coordinate transformations. We have furthermore used the abbreviations $\mathrm{NC}$ and $\mathrm{NH}$.

\begin{tabular}{lcccc}
\hline \hline Background fields & Particle $\Lambda=0$ & Symmetries $\Lambda=0$ & Particle $\Lambda \neq 0$ & Symmetries $\Lambda \neq 0$ \\
\hline None & Galilean & Galilei & NH & NH \\
$\Phi$ & Curved Galilean & a.e. Galilei & Curved NH & a.e. Galilei \\
$e_{\mu}^{a}, \tau_{\mu}, m_{\mu}$ & NC & g.c.t. & NC NH & g.c.t. \\
\hline \hline
\end{tabular}

$\kappa$-symmetry corresponds to a fermionic gauge shift symmetry, i.e. a Stückelberg symmetry [15].

When discussing the symmetries of (super)particles in a curved background it is important to distinguish between "proper" and "sigma model" symmetries [29,30]. In the case of proper symmetries the background (super)gravity fields only transform through their dependence on the embedding coordinates of the (super)particle. On the other hand, in the case of sigma model symmetries the background fields have their own transformation rules. We will clarify how these sigma model transformations are related to the transformations of the (super)gravity fields when viewed as the components of a (super)gravity multiplet defined in the target space.

To explain our construction of nonrelativistic superparticles in a curved background, we will first consider the bosonic case and take as our starting point a single massive nonrelativistic particle in a flat background. The action of such a particle is invariant under the (global) Galilei symmetries, hence the name "Galilean" particle. We next partially gauge the spatial target space translations of the Galilean particle such that the constant parameter of a spatial translation is promoted to an arbitrary function of time. The resulting extended symmetries are sometimes called the "acceleration-extended" Galilean symmetries. To achieve this partial gauging, the particle must move in a curved Galilean background that is characterized by the Newton potential $\Phi[31] .^{2}$ We will call this particle a "curved Galilean" particle. Finally, we perform a full gauging of the Galilean symmetries such that the parameter of spatial translations becomes an arbitrary function of the spacetime coordinates and the full symmetries of the particle action are the general coordinate transformations. Actually, to perform this gauging it is necessary to extend the Galilei symmetries with an additional central charge transformation $^{3} \quad[32,35,36]$. The background is now

\footnotetext{
${ }^{2}$ There is also a different way of gauging the spatial translations where the background is given by a vector field rather than a scalar potential $[32,33]$.

${ }^{3}$ In Secs. III and IV, where we discuss supersymmetric particle actions, we will restrict ourselves to $d=3$. In that case, it is known that the Bargmann algebra admits a second central charge; see e.g. Ref. [34]. We will however not consider it in this paper.
}

promoted to a Newton-Cartan gravity background that is characterized by a spacelike Vielbein $e_{\mu}{ }^{a}$, a timelike Vielbein $\tau_{\mu}$ and a central charge gauge field $m_{\mu}$. We will call the corresponding particle a "Newton-Cartan" (NC) particle. In Table I we have summarized the three different situations described above.

In this work we will extend the superparticle actions considered so far in two directions. First of all, we will deform the different backgrounds with a cosmological constant $\Lambda$. For a flat ${ }^{4}$ background the Galilei symmetries are then deformed to so-called Newton-Hooke (NH) symmetries $[38,39] .{ }^{5}$ We will call the corresponding particle a "Newton-Hooke" particle. The cosmological extensions of the curved Galilean and NC particles are called the curved $\mathrm{NH}$ and $\mathrm{NC} \mathrm{NH}$ particles, respectively. It turns out that the symmetries corresponding to these particle actions are the same as in the absence of a cosmological constant; see Table I. The reason for this is that the Galilean and NH symmetries only differ in specific spatial translations which all become part of the same timedependent translations (curved Galilean and curved $\mathrm{NH}$ particles) or spacetime-dependent translations ( $\mathrm{NC}$ and $\mathrm{NC}$ $\mathrm{NH}$ particles). The three different cases with $\Lambda \neq 0$ are indicated in the last two columns of Table I.

The second extension we will consider is the one from the bosonic particle to the superparticle. This requires a supersymmetric extension of the gravity backgrounds in the first place. Since nonrelativistic supergravity multiplets to our knowledge have only been explicitly constructed in three dimensions, we will only consider superparticles in a three-dimensional (3D) background. A supersymmetric version of the 3D Galilean and $\mathrm{NC}$ backgrounds was recently constructed by gauging the Galilei, or better Bargmann, superalgebra [47]. We will make full use of the construction of Ref. [47] which, in particular, explains how to switch between different backgrounds, with different symmetries, by partial gauging or partial gauge fixing. Our aim will be to investigate the action of a 3D

\footnotetext{
${ }^{4}$ Note that after adding the cosmological constant space is flat while spacetime is curved, see e.g. [37].

${ }^{5}$ For earlier works on NH symmetries including their supersymmetric extension; see e.g. Refs. [16,40-46].
} 


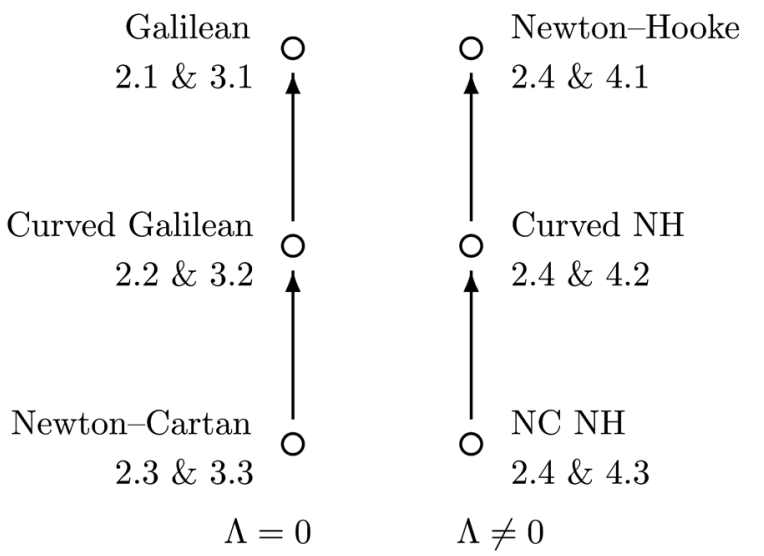

FIG. 1. This figure displays the different backgrounds used in this paper and the sections where they are discussed. For each background, the first section discusses the bosonic case while the second section treats the supersymmetric case. The arrows indicate the direction of gauge fixing. From left to right we turn on a cosmological constant $\Lambda$.

superparticle first in a flat background and, next, in a Galilean and NC supergravity background with and without a cosmological constant. To indicate the different cases we will use the same nomenclature as in the bosonic case but with the word particle replaced by superparticle.

This work is organized as follows. In Sec. II we go through the known descriptions of the bosonic particle to introduce our notation and discuss the different gaugings and gauge fixings in a simple setting. After discussing the $\Lambda=0$ case in some detail we repeat the analysis for a nonzero cosmological constant. Sections III and IV are devoted to the supersymmetrization of the theories discussed in Sec. II without and with a nonzero cosmological constant, respectively. Section V is reserved for some comments on the role of $\kappa$-symmetry in the nonrelativistic case. A few technicalities, that are used in the main text, are relegated to three Appendices. Finally, we have given an alternative table of contents of this paper in Fig. 1 which summarizes in which sections we discuss the different backgrounds that we will consider.

\section{NONRELATIVISTIC BOSONIC PARTICLE}

This section will serve as an introduction as well as a means to familiarize the reader with our notation. Moreover, it is a useful warmup exercise for the rest of the paper when we discuss the superparticle. In Sec. II A we start by discussing the bosonic Galilean particle in a flat spacetime without gravitational dynamics. Next, in Sec. II B we will partially gauge the Galilei symmetries to allow for arbitrary time-dependent translations. This introduces the curved Galilean particle which has gravitational dynamics described by a Newton potential; see Ref. [48]. In Sec. II C we will perform a further gauging and obtain the $\mathrm{NC}$ particle action which is invariant under general coordinate transformations. Finally, in Sec. II D, we repeat the analysis of the previous three subsections in the presence of a cosmological constant.

\section{A. Galilean particle}

We consider a bosonic particle moving in a flat $d$-dimensional background with embedding coordinates $\left\{t(\tau), x^{i}(\tau)\right\}, i=1, \ldots, d-1$, with $\tau$ the evolution parameter. The action describing the dynamics of this bosonic particle is given by

$$
S=\int d \tau \frac{m}{2} \frac{\dot{x}^{i} \dot{x}^{i}}{\dot{t}}
$$

The dot indicates a differentiation with respect to the evolution parameter $\tau$. The equations of motion for $t$ and $x^{i}$ corresponding to the action (2.1) are not independent. The first can be derived from the latter which is given by

$$
\frac{d}{d \tau}\left(\frac{\dot{x}^{i}}{\dot{t}}\right)=0
$$

This implies the existence of a gauge transformation. In fact, the action (2.1) is invariant under worldline reparameterizations with parameter $\rho(\tau){ }^{6}$

$$
\delta t=\rho(\tau) \dot{t}, \quad \delta x^{i}=\rho(\tau) \dot{x}^{i},
$$

together with the Galilei symmetries

$$
\delta t=-\zeta, \quad \delta x^{i}=\lambda^{i}{ }_{j} x^{j}-v^{i} t-a^{i} .
$$

Here, $\left(\zeta, \lambda^{i}{ }_{j}, v^{i}, a^{i}\right)$ parametrize a (constant) time translation, spatial rotation, boost transformation and space translation, respectively. Note that the Lagrangian (2.1) is invariant under boosts only up to a total $\tau$-derivative. This has the consequence that the algebra of Nöther charges is given by a centrally extended Galilei algebra which is called the Bargmann algebra $[49,50]$. The transformations (2.4) are a representation of the Galilean algebra

$$
\begin{array}{ll}
{\left[J_{a b}, P_{c}\right]=-2 \delta_{c[a} P_{b]},} & {\left[G_{a}, H\right]=-P_{a},} \\
{\left[J_{a b}, G_{c}\right]=-2 \delta_{c[a} G_{b]},} & {\left[J_{a b}, J_{c d}\right]=4 \delta_{[a[c} J_{d] b]},}
\end{array}
$$

where the generators

$$
\left\{H, J_{a b}, P_{a}, G_{a}\right\}
$$

generate time translations, spatial rotations, space translations and boost transformations, respectively. The Bargmann algebra has the additional commutation relation

\footnotetext{
${ }^{6}$ In the following we refrain from denoting these worldline reparameterizations explicitly.
} 


$$
\left[P_{a}, G_{b}\right]=\delta_{a b} Z
$$

where $Z$ is the generator of central charge transformations. Physically, the occurrence of the central charge transformations is related to the fact that at the nonrelativistic level the mass of a particle is conserved.

\section{B. Curved Galilean particle}

We next consider the curved Galilean particle, i.e. a bosonic particle in a Galilean gravity background described by a Newton potential $\Phi(t, \vec{x})$. The action for the curved Galilean particle can be derived by gauging the spatial translations (2.4) to allow for arbitrary time-dependent boost parameters $\xi^{i}(t)$. This extension of the Galilean symmetries is called the acceleration-extended Galilei symmetries or the Milne symmetries [51]. As explained in Ref. [48] this gauging procedure leads to the following action:

$$
S=\int d \tau \frac{m}{2}\left[\frac{\dot{x}^{i} \dot{x}^{i}}{\dot{t}}-2 \dot{t} \Phi\right]
$$

The action (2.8) is invariant under the worldline reparameterizations (2.3) and under the acceleration-extended symmetries

$$
\delta t=-\zeta, \quad \delta x^{i}=\lambda_{j}^{i} x^{j}-\xi^{i}(t) .
$$

The acceleration-extended symmetries are not a proper symmetry of the action (2.8). Instead, the Newton potential should be viewed as a background field and the acceleration-extended symmetries as sigma model symmetries. In particular, the transformation rule of the background field, that we will denote by the symbol $\delta_{\text {bg }}$, lacks the transport terms that are present in the transformation rule associated to a proper symmetry, denoted in this paper by $\delta_{\mathrm{pr}}{ }^{7}$ :

$$
\delta_{\mathrm{bg}}=\delta_{\mathrm{pr}}+\delta x^{\mu} \partial_{\mu}
$$

Using this we find that the action (2.8) is invariant under the acceleration-extended symmetries (2.9) provided the Newton potential $\Phi$ transforms as follows:

$$
\delta_{\mathrm{bg}} \Phi=\frac{1}{\dot{t}} \frac{d}{d \tau}\left(\frac{\dot{\xi}^{i}}{\dot{t}}\right) x^{i}+\sigma(t) .
$$

The second term with the arbitrary function $\sigma(t)$ represents a standard ambiguity in any potential describing a force and gives a boundary term in the action (2.8). An important

\footnotetext{
${ }^{7}$ Assuming that $x^{\mu} \rightarrow x^{\mu}+\delta x^{\mu}$ a transport term is given by $-\delta x^{\mu} \partial_{\mu}$ so that the second term in (2.10) cancels the transport term present in the proper transformation rule represented by the first term.
}

qualification of the background field is that it has to obey the constraint

$$
\partial^{i} \partial_{i} \Phi=0
$$

We will refer to this constraint as the equation of motion of the background field.

We can reobtain the particle in flat space by setting

$$
\Phi=0 .
$$

This reduces the action and transformation rules of the curved Galilean particle to the one of the Galilean particle given in the previous subsection.

\section{Newton-Cartan particle}

We now wish to extend the curved Galilean particle to a $\mathrm{NC}$ particle, i.e. a particle moving in a NC gravity background and invariant under general coordinate transformations. To describe a NC background we need a temporal Vielbein $\tau_{\mu}$ and a spatial Vielbein $e_{\mu}{ }^{a}, a=1, \ldots, d-1$, We furthermore need to introduce a central charge gauge field $m_{\mu}$. The action for the NC particle was already derived in Ref. [52], see also Ref. [48], and is given by

$$
S=\int d \tau \frac{m}{2}\left[\frac{\dot{x}^{\mu} e_{\mu}{ }^{a} \dot{x}^{\nu} e_{\nu a}}{\dot{x}^{\rho} \tau_{\rho}}-2 m_{\mu} \dot{x}^{\mu}\right] .
$$

This action is invariant under the worldline reparameterizations (2.3) and under the gauged Galilei symmetries, i.e. general coordinate transformations. The embedding coordinates transform under these general coordinate transformations, with parameters $\xi^{\mu}\left(x^{\nu}\right)$, in the standard way:

$$
\delta x^{\mu}=-\xi^{\mu}\left(x^{\nu}\right) .
$$

The transformation rules of the background fields $\tau_{\mu}, e_{\mu}{ }^{a}$ and $m_{\mu}$ follow from the known proper transformation rules by omitting the transport term, see Eq. (2.10):

$$
\begin{aligned}
\delta_{\mathrm{bg}} \tau_{\mu} & =\partial_{\mu} \xi^{\rho} \tau_{\rho}, \\
\delta_{\mathrm{bg}} e_{\mu}{ }^{a} & =\partial_{\mu} \xi^{\rho} e_{\rho}{ }^{a}+\lambda^{a}{ }_{b} e_{\mu}{ }^{b}+\lambda^{a} \tau_{\mu}, \\
\delta_{\mathrm{bg}} m_{\mu} & =\partial_{\mu} \xi^{\rho} m_{\rho}+\partial_{\mu} \sigma+\lambda_{a} e_{\mu}{ }^{a} .
\end{aligned}
$$

Here, $\lambda_{b}^{a}, \lambda^{a}$ and $\sigma$ are the parameters of a local spatial rotation, boost transformation and central charge transformation, respectively.

The proper transformation rules of the background fields $\tau_{\mu}, e_{\mu}{ }^{a}$ and $m_{\mu}$ can be obtained by gauging the Bargmann algebra; see e.g. Ref. [36]. Since the NC background is the most general background one must be able to obtain the transformations of the curved Galilean and flat backgrounds discussed in the two previous subsections by gauge fixing some of the general coordinate transformations. This is discussed in detail in Ref. [47]. For the 
convenience of the reader, we list in Table II the gaugefixing conditions that need to be imposed on the $\mathrm{NC}$ background fields, and the compensating gauge transformations that come along with it, that bring us to the curved Galilean background in terms of the Newton potential $\Phi$.

\section{Adding a cosmological constant (bosonic)}

We now extend the analysis and consider particles in a background with a cosmological constant $\Lambda$. We only consider the case of a negative cosmological constant. One way to obtain the action and transformation rules is by using the method of nonlinear realizations [53,54] applied to the nonrelativistic contraction of the (anti-)de Sitter [(A)dS] algebra which is the so-called NewtonHooke algebra. In the supersymmetric case this is done in Appendix B. The corresponding Newton-Hooke superalgebra is given in Appendix A. As we will see below, in the bosonic case an easier way of getting a cosmological extension is to take the nonrelativistic limit, as described e.g. in Ref. [48], of the relativistic particle action in an (A) $\mathrm{dS}$ background. Below we will discuss the cosmological extension of the Galilean, curved Galilean and NC particles, one after the other. Following the nomenclature given in Table I, this will lead to the $\mathrm{NH}$, curved $\mathrm{NH}$ and $\mathrm{NC} \mathrm{NH}$ particles, respectively.

\section{Newton-Hooke particle}

We consider a negative cosmological constant $\Lambda<0$ with the AdS radius given by $R^{2}=-1 / \Lambda^{8}$ In global coordinates the metric of an AdS spacetime is given by

$d s^{2}=-f(r) d t^{2}+\frac{1}{f(r)} d r^{2}+r^{2} d \varphi^{2}, \quad f(r)=1-\Lambda r^{2}$.

By taking the nonrelativistic limit of a relativistic particle in such an AdS background we arrive at the action

$$
S=\int d \tau \frac{m}{2}\left[\frac{\dot{x}^{i} \dot{x}^{i}}{\dot{t}}-\frac{\dot{t} x^{i} x^{i}}{R^{2}}\right],
$$

once a total derivative term is eliminated. Physically, this system is equivalent to the nonrelativistic harmonic oscillator. This action is invariant under a deformation of the Galilei transformations (2.4). In particular, the transformations under boosts and spatial translations are given by

$$
\delta x^{i}=-v^{i} R \sin \frac{t}{R}-a^{i} \cos \frac{t}{R}
$$

\footnotetext{
${ }^{8}$ In the following we will express everything in terms of the radius $R$.
}

The other Galilei transformations are not deformed. The symmetry algebra is the $\mathrm{NH}$ algebra, which consists of the Galilei algebra (2.5) plus the additional commutator

$$
\left[P_{a}, H\right]=\frac{1}{R^{2}} G_{a} .
$$

In the limit $R \rightarrow \infty$ the action, transformation rules and the algebra reduce to those of the Galilean particle.

\section{Curved Newton-Hooke particle}

We can partially gauge the symmetries of the $\mathrm{NH}$ particle in the same way as we did in the case of the Galilean particle, see Sec. II B, by introducing a gravitational potential $\phi$. The resulting action is given by

$$
S=\int d \tau \frac{m}{2}\left[\frac{\dot{x}^{i} \dot{x}^{i}}{\dot{t}}-\dot{t} \frac{x^{i} x^{i}}{R^{2}}-2 \dot{t} \phi\right]
$$

It is invariant under the acceleration-extended symmetries (2.9), but the transformation of the background field changes:

$$
\delta_{\mathrm{bg}} \phi=\frac{1}{\dot{t}} \frac{d}{d \tau}\left(\frac{\dot{\xi}^{i}}{\dot{t}}\right) x^{i}+\frac{\xi^{i}(t) x^{i}}{R^{2}}+\sigma(t) .
$$

The explicit $1 / R$ terms that appear in the action (2.21) and the transformation rule (2.22) are due to our choice of the gravitational potential $\phi$. Namely, we demand that the equation of motion of the potential is $\partial^{i} \partial_{i} \phi=0 .^{9}$

Setting the gravitational potential $\phi$ to zero leads us back to the NH particle. The differential equation that follows from Eq. (2.22) then fixes the time dependence of $\xi^{i}(t)$ to be the one given in Eq. (2.19).

\section{Newton-Cartan Newton-Hooke particle}

A cosmological extension of the $\mathrm{NC}$ particle discussed in Sec. IIC can be obtained along the same lines as we discussed for the curved $\mathrm{NH}$ particle above. The following action is obtained:

$S=\int d \tau \frac{m}{2}\left[\frac{\dot{x}^{\mu} e_{\mu}{ }^{a} \dot{x}^{\nu} e_{\nu a}}{\dot{x}^{\rho} \tau_{\rho}}-2 m_{\mu} \dot{x}^{\mu}-\dot{x}^{\rho} \tau_{\rho} \frac{x^{\mu} e_{\mu}{ }^{a} x^{\nu} e_{\nu a}}{R^{2}}\right]$

\footnotetext{
${ }^{9}$ Note that we could simplify the formulas (2.21) and (2.22) by defining a new potential$$
\tilde{\Phi}=\phi+\frac{x^{i} x^{i}}{2 R^{2}}
$$

This would remove all $1 / R$ terms in (2.21) and (2.22), but it would also alter the equation of motion. By our definition $\tilde{\Phi}$ would not be a gravitational potential. Nevertheless, for technical reasons it is useful to define such a shifted potential, especially in the supersymmetric case.
} 
TABLE II. This table indicates the gauge-fixing conditions, chronologically ordered from top to bottom, and the corresponding compensating transformations, that lead from the NC particle, see Sec. II C, to the curved Galilean particle, see Sec. II B. Note that $\tau_{i}\left(x^{\nu}\right) \equiv e_{i}{ }^{0}\left(x^{\nu}\right)$. The restriction $\tau_{i}\left(x^{\nu}\right)+m_{i}\left(x^{\nu}\right)=\partial_{i} m\left(x^{\nu}\right)$ follows from the gauge conditions made at that point.

\begin{tabular}{lc}
\hline \hline Gauge condition & Compensating transformations \\
\hline$\tau_{\mu}\left(x^{\nu}\right)=\delta_{\mu}{ }^{\emptyset}$ & $\xi^{\emptyset}\left(x^{\nu}\right)=\xi^{\emptyset}$ \\
$\omega_{\mu}{ }^{a b}=0$ & $\lambda^{a b}\left(x^{\nu}\right)=\lambda^{a b}$ \\
$e_{i}{ }^{a}=\delta_{i}{ }^{a}$ & $\xi^{i}\left(x^{\nu}\right)=\xi^{i}(t)-\lambda^{i}{ }_{j} x^{j}$ \\
$\tau_{i}\left(x^{\nu}\right)+m_{i}\left(x^{\nu}\right)=\partial_{i} m\left(x^{\nu}\right)$ & \\
$m\left(x^{\nu}\right)=0$ & $\sigma\left(x^{\nu}\right)=\sigma(t)+\partial_{t} \xi^{i}(t) x^{i}$ \\
$\tau_{i}\left(x^{\nu}\right)=0$ & $\lambda^{i}\left(x^{\nu}\right)=-\partial_{t} \xi^{i}(t)$ \\
$m_{\varnothing}\left(x^{\nu}\right)=\Phi\left(x^{\nu}\right)$ & $\omega_{\varnothing}{ }^{a}=-\partial_{a} \Phi\left(x^{\nu}\right)$ \\
\hline \hline
\end{tabular}

This action is invariant under the gauged Galilei symmetries (which are equivalent to the gauged $\mathrm{NH}$ symmetries). The transformations of the background fields $\tau_{\mu}$ and $e_{\mu}{ }^{a}$ are given by Eq. (2.16), while the transformation of the central charge gauge field $m_{\mu}$ reads

$$
\begin{aligned}
\delta_{\mathrm{bg}} m_{\mu}= & \partial_{\mu} \sigma+\lambda^{a} e_{\mu a}-\tau_{\mu} \frac{x^{\rho} e_{\rho a} x^{\nu}}{R^{2}} \lambda^{a} \tau_{\rho} \\
& +m_{\rho} \partial_{\mu} \xi^{\rho}+\tau_{\mu} \frac{x^{\nu} e_{\nu}{ }^{a}}{R^{2}} \xi^{\rho} e_{\rho}{ }^{a}-\tau_{\mu} \frac{x^{\nu} e_{\nu}{ }^{a}}{R^{2}} x^{\rho} e_{\alpha}{ }^{a} \partial_{\rho} \xi^{\alpha} .
\end{aligned}
$$

By imposing the gauge-fixing conditions enlisted in Table II one obtains the action and symmetries of the curved NH particle; see Eqs. (2.21) and (2.22).

This finishes our discussion of the bosonic particle in different backgrounds. In the remaining part of this work we will discuss the supersymmetric extension of these different particles and backgrounds.

\section{NONRELATIVISTIC SUPERPARTICLE}

In the same way that the nonrelativistic bosonic particle is based upon the Galilei algebra, or its centrally extended version, the Bargmann algebra, the action and transformation rules of the nonrelativistic $3 \mathrm{D} \mathcal{N}=2$ superparticle are based upon the supersymmetric extension of the Galilei or Bargmann algebra. It turns out that we need two supersymmetries since one of the supersymmetries is, like the time translations in the bosonic case, a Stückelberg symmetry.

The 3D $\mathcal{N}=2$ Galilei superalgebra is given by the 3D version of the bosonic commutation relations (2.5) plus the additional relations $[25,26]$

$$
\begin{aligned}
{\left[J_{a b}, Q^{ \pm}\right] } & =-\frac{1}{2} \gamma_{a b} Q^{ \pm}, \quad\left[G_{a}, Q^{+}\right]=-\frac{1}{2} \gamma_{a 0} Q^{-}, \\
\left\{Q_{\alpha}^{+}, Q_{\beta}^{+}\right\} & =2\left[\gamma^{0} C^{-1}\right]_{\alpha \beta} H, \quad\left\{Q_{\alpha}^{+}, Q_{\beta}^{-}\right\}=\left[\gamma^{a} C^{-1}\right]_{\alpha \beta} P_{a},
\end{aligned}
$$

where the Majorana spinors $Q_{\alpha}^{ \pm}$are the generators corresponding to the two supersymmetries. ${ }^{10}$ In the case of the 3D $\mathcal{N}=2$ Bargmann superalgebra there is a central extension given by Eq. (2.7) and by the following equation:

$$
\left\{Q_{\alpha}^{-}, Q_{\beta}^{-}\right\}=2\left[\gamma^{0} C^{-1}\right]_{\alpha \beta} Z \text {. }
$$

The 3D $\mathcal{N}=2$ supergravity background has been obtained in Ref. [47] by gauging the algebra above. This result allows us to discuss the nonrelativistic superparticle for $\Lambda=0$, i.e. the supersymmetric version of the second column of Table I. In the three subsections below we will discuss the Galilean, the curved Galilean and the NC superparticles, respectively.

In the relativistic case the superparticle has an additional so-called $\kappa$-symmetry. In the nonrelativistic analog this becomes a Stuickelberg symmetry [15]. For the purpose of this section we will fix $\kappa$-symmetry to avoid unnecessary cluttering of our formulas. Some remarks about restoring $\kappa$ symmetry are separately given in Sec. V.

\section{A. Galilean superparticle}

The Galilean superparticle was already discussed in Ref. [15]. In terms of the bosonic and fermionic embedding coordinates $\left\{t, x^{i}, \theta_{-}\right\}$the action is given by

$$
S=\int d \tau \frac{m}{2}\left[\frac{\dot{x}^{i} \dot{x}^{i}}{\dot{t}}-\bar{\theta}_{-} \gamma^{0} \dot{\theta}_{-}\right]
$$

This action is invariant under the following Galilei symmetries:

$\delta t=-\zeta, \quad \delta x^{i}=\lambda^{i}{ }_{j} x^{j}-v^{i} t-a^{i} . \quad \delta \theta_{-}=\frac{1}{4} \lambda^{a b} \gamma_{a b} \theta_{-}$.

The same action is invariant under two supersymmetries with constant parameters $\epsilon_{+}$and $\epsilon_{-}$:

$\delta t=0, \quad \delta x^{i}=-\frac{1}{2} \bar{\epsilon}_{+} \gamma^{i} \theta_{-}, \quad \delta \theta_{-}=\epsilon_{-}-\frac{\dot{x}^{i}}{2 \dot{t}} \gamma_{0 i} \epsilon_{+}$.

One may verify that the above set of transformation rules (3.4) and (3.5) closes off shell. Note that the transformation with parameter $\epsilon_{+}$is realized linearly. Instead, the one with parameter $\epsilon_{-}$is realized nonlinearly; i.e. it is a broken symmetry. This implies that the superparticle corresponds to a $1 / 2$ Bogomol'nyi-Prasad-Sommerfield (BPS) state.

\footnotetext{
${ }^{10} \mathrm{We}$ use a Majorana representation in which the charge conjugation matrix is given by $C=i \gamma^{0}$ and all $\gamma$-matrices are real, i.e. $\gamma^{\mu}=\left(i \sigma_{2}, \sigma_{1}, \sigma_{3}\right)$.
} 


\section{B. Curved Galilean superparticle}

We will now extend the Galilean superparticle to a curved Galilean superparticle thereby replacing the flat background by a Galilean supergravity background. This corresponds to extending the bosonic particle in a Galilean gravity background, discussed in Sec. II B, to the supersymmetric case.

Our starting point is the superparticle action in a flat background; see Eq. (3.3). We will now partially gauge the transformations (3.4) and (3.5) to allow for arbitrary timedependent boosts, with parameters $\xi^{i}(t)$, and arbitrary supersymmetry transformations, with parameters $\epsilon_{-}(t)$. The complete bosonic and fermionic transformation rules now read

$\delta t=-\zeta, \quad \delta x^{i}=\lambda^{i}{ }_{j} x^{j}-\xi^{i}(t), \quad \delta \theta_{-}=\frac{1}{4} \lambda^{a b} \gamma_{a b} \theta_{-}$,

and

$\delta t=0, \quad \delta x^{i}=-\frac{1}{2} \bar{\epsilon}_{+} \gamma^{i} \theta_{-}, \quad \delta \theta_{-}=\epsilon_{-}(t)-\frac{\dot{x}^{i}}{2 \dot{t}} \gamma_{0 i} \epsilon_{+}$,

respectively.

The Galilean supergravity multiplet, that we will use to perform the partial gauging of the transformations (3.4) and (3.5), was introduced in Ref. [47]. Alongside the Newton potential $\Phi(x)$, it also contains a fermionic background field $\Psi(x)$. The equations of motion for these two background fields are

$$
\partial^{i} \partial_{i} \Phi=0, \quad \gamma^{i} \partial_{i} \Psi=0 .
$$

There is a slight subtlety regarding this Galilean supergravity multiplet, stemming from the fact that $\Psi(x)$ is the superpartner of the Newton force $\Phi_{i} \equiv \partial_{i} \Phi(x)$ and not of the Newton potential itself. The transformation rules of the Newton force are, however, compatible with the integrability condition $\partial_{[i} \Phi_{j]}=0$, so that they can be integrated to transformation rules of the Newton potential $\Phi(x)$. This is done via the introduction of a fermionic prepotential $\chi(x)$, that will be called the "Newtino potential," defined via

$$
\partial_{i} \chi=\gamma_{i} \Psi(i=1,2), \quad \gamma^{1} \partial_{1} \chi=\gamma^{2} \partial_{2} \chi,
$$

where the second equation represents a constraint obeyed by $\chi(x)$, as a consequence of its definition. This constraint can be interpreted (upon choosing a specific basis for the $\gamma$ matrices) as the Cauchy-Riemann equations, expressing holomorphicity of $\chi_{1}+\mathrm{i} \chi_{2}$, where $\chi_{1,2}$ are the components of $\chi$. Since the Newton potential $\Phi$ obeys the Laplace equation in two spatial dimensions, it can also be seen as the real part of a holomorphic function $\Phi+\mathrm{i} \Xi$. The imaginary part $\Xi(x)$ of this function was called the "dual Newton potential" in Ref. [47] and is related to $\Phi(x)$ via the Cauchy-Riemann equations for $\Phi+\mathrm{i} \Xi$ :

$$
\partial_{i} \Phi=\varepsilon_{i j} \partial^{j} \Xi, \quad \partial_{i} \Xi=-\varepsilon_{i j} \partial^{j} \Phi .
$$

The dual Newton potential was introduced in Ref. [47] in order to write down the supersymmetry transformation rule for $\chi$. Since this is a transformation rule for both real and imaginary parts of $\chi_{1}+\mathrm{i} \chi_{2}$, it is natural to expect that it involves also both real and imaginary parts of $\Phi+\mathrm{i} \Xi$, and this is indeed the case.

We find that the action of the curved Galilean superparticle in terms of the Galilean supergravity background fields $\Phi$ and $\Psi$ is given by

$S=\int d \tau \frac{m}{2}\left[\frac{\dot{x}^{i} \dot{x}^{i}}{\dot{t}}-\bar{\theta}_{-} \gamma^{0} \dot{\theta}_{-}-2 \dot{t} \Phi+2 \dot{t} \bar{\theta}_{-} \gamma^{0} \Psi\right]$.

One may verify that the action (3.11) is invariant under the transformations (3.6) and (3.7) provided that the background fields transform under the bosonic symmetries as

$\delta_{\mathrm{bg}} \Phi=\frac{1}{\dot{t}} \frac{d}{d \tau}\left(\frac{\dot{\xi}^{i}}{\dot{t}}\right) x^{i}+\sigma(t), \quad \delta_{\mathrm{bg}} \Psi=\frac{1}{4} \lambda^{a b} \gamma_{a b} \Psi$

and under the fermionic symmetries as

$$
\begin{gathered}
\delta_{\text {bg }} \Phi=\bar{\epsilon}_{-} \gamma^{0} \Psi+\frac{1}{2} \bar{\epsilon}_{+} \partial_{t} \chi-\frac{1}{2} \bar{\epsilon}_{+} \gamma^{i} \theta_{-} \partial_{i} \Phi, \\
\delta_{\text {bg }} \Psi=\frac{1}{\dot{t}} \dot{\epsilon}_{-}-\frac{1}{2} \partial_{i} \Phi \gamma_{i 0} \epsilon_{+}-\frac{1}{2} \bar{\epsilon}_{+} \gamma^{i} \theta_{-} \partial_{i} \Psi .
\end{gathered}
$$

The only invariance that is nontrivial to show is the one under the linear $\epsilon_{+}$transformations. Varying the action (3.11) under $\epsilon_{+}$-transformations one is left with the following terms:

$\delta_{+} S=\int d \tau \frac{m}{2}\left[-\bar{\epsilon}_{+} \dot{t} \partial_{t} \chi-\bar{\epsilon}_{+} \dot{x}^{i} \partial_{i} \chi-\frac{\dot{t}}{2} \bar{\epsilon}_{+} \gamma^{k} \theta_{-} \bar{\theta}_{-} \gamma^{0 i} \partial_{i} \partial_{k} \chi\right]$.

The first two terms combine into a total $\tau$-derivative, since $\chi$ is a function of $x^{i}$ and $t$ and therefore

$$
\frac{d}{d \tau} \chi=\left(\dot{t} \partial_{t}+\dot{x}^{i} \partial_{i}\right) \chi .
$$

The second term vanishes upon using the equation of motion for the background field $\chi$.

To calculate the commutator algebra it is important to keep in mind that the background fields do not transform as fundamental fields but, instead, according to background 
fields; see Eq. (2.10). This explains the "wrong" sign transport term in the $\epsilon_{+}$transformation and the absence of transport terms for all other symmetries. It also has the consequence that partial derivatives do not commute with background variations:

$$
\left[\delta_{\mathrm{bg}}, \partial_{\mu}\right]=-\left(\partial_{\mu} \delta x^{\nu}\right) \partial_{\nu} .
$$

Another subtlety when calculating the commutator algebra is related to the fact that the parameters $\xi^{i}$ and $\epsilon_{-}$are functions of the time $t$ but that $t$ itself is a scalar function $t(\tau)$ of the worldline parameter $\tau$. This implies that when we calculate commutators we have to vary the $t$ inside the parameters. Keeping the above subtleties in mind we find that the commutation relations close off shell on the embedding coordinates and the background fields.

Imposing the gauge-fixing conditions

$$
\Phi=1, \quad \chi=0,
$$

we recover the Galilean superparticle with the flat spacetime transformation rules (3.4) and (3.5). Imposing the additional gauge-fixing condition

$$
t=\tau
$$

we find agreement with the algebra obtained in Ref. [47].

\section{Newton-Cartan superparticle}

We wish to extend the result of the previous subsection to arbitrary frames corresponding to a superparticle in a Newton-Cartan supergravity background. Due to the complexity of the calculations we only give the result up to quartic fermions in the action. We find that using this approximation the action is given by

$$
\begin{aligned}
S= & \int d \tau \frac{m}{2}\left[\frac{\dot{x}^{\mu} e_{\mu}{ }^{a} \dot{x}^{\nu} e_{\nu a}}{\dot{x}^{\rho} \tau_{\rho}}-2 m_{\mu} \dot{x}^{\mu}-\bar{\theta}_{-} \gamma^{0} D_{\tau} \theta_{-}\right. \\
& \left.+2 \bar{\theta}_{-} \gamma^{0} \psi_{\mu-} \dot{x}^{\mu}-\frac{\dot{x}^{\mu} e_{\mu a}}{\dot{x}^{\rho} \tau_{\rho}} \bar{\theta}_{-} \gamma^{a} \psi_{\nu+} \dot{x}^{\nu}\right],
\end{aligned}
$$

where the Lorentz-covariant derivative $D_{\tau}$ is defined as

$$
D_{\tau} \theta_{-}=\dot{\theta}_{-}-\frac{1}{4} \dot{x}^{\mu} \omega_{\mu}^{a b} \gamma_{a b} \theta_{-} .
$$

To lowest order in the fermions the action (3.20) is invariant under the following bosonic and fermionic symmetries of the embedding coordinates:

$$
\delta x^{\mu}=-\xi^{\mu}\left(x^{\alpha}\right), \quad \delta \theta_{-}=\frac{1}{4} \lambda^{a b}\left(x^{\alpha}\right) \gamma_{a b} \theta_{-},
$$

and

$$
\begin{aligned}
\delta x^{\mu} & =-\frac{1}{2} \bar{\epsilon}_{+}\left(x^{\alpha}\right) \gamma^{a} \theta_{-} e^{\mu}{ }_{a}, \\
\delta \theta_{-} & =\epsilon_{-}\left(x^{\alpha}\right)-\frac{\dot{x}^{\mu} e_{\mu}{ }^{a}}{2 \dot{x}^{\rho} \tau_{\rho}} \gamma_{0 a} \epsilon_{+}\left(x^{\alpha}\right) .
\end{aligned}
$$

In the following we refrain from explicitly denoting the local $x^{\mu}$ dependence of the parameters.

The transformation rules of the background fields follow from the supergravity result given in Ref. [47] and application of the identity (2.10). We find that the bosonic transformation rules are given by

$$
\begin{aligned}
\delta_{\mathrm{pr}} \tau_{\mu} & =0, \quad \delta_{\mathrm{pr}} m_{\mu}=\partial_{\mu} \sigma+\lambda_{a} e_{\mu}{ }^{a}, \\
\delta_{\mathrm{pr}} e_{\mu}{ }^{a} & =\lambda^{a}{ }_{b} e_{\mu}{ }^{b}+\lambda^{a} \tau_{\mu}, \quad \delta_{\mathrm{pr}} \psi_{\mu+}=\frac{1}{4} \lambda^{a b} \gamma_{a b} \psi_{\mu+}, \\
\delta_{\mathrm{pr}} \omega_{\mu}{ }^{a b} & =\partial_{\mu} \lambda^{a b}, \quad \delta_{\mathrm{pr}} \psi_{\mu-}=\frac{1}{4} \lambda^{a b} \gamma_{a b} \psi_{\mu-}-\frac{1}{2} \lambda^{a} \gamma_{a 0} \psi_{\mu+}, \\
\delta_{\mathrm{pr}} \omega_{\mu}{ }^{a} & =\partial_{\mu} \lambda^{a}-\lambda_{b} \omega_{\mu}{ }^{a b}+\lambda^{a b} \omega_{\mu b} .
\end{aligned}
$$

To keep the formulas simple we have given here as well as below only the proper transformation rules. The background transformations are obtained by supplementing each of these rules with an additional transformation under general coordinate transformations; see Eq. (2.10). For the fermionic transformations we find the following expressions:

$$
\begin{aligned}
\delta_{\mathrm{pr}} \tau_{\mu} & =\frac{1}{2} \bar{\epsilon}_{+} \gamma^{0} \psi_{\mu+}, \quad \delta_{\mathrm{pr}} m_{\mu}=\bar{\epsilon}_{-} \gamma^{0} \psi_{\mu-}, \\
\delta_{\mathrm{pr}} e_{\mu}{ }^{a} & =\frac{1}{2} \bar{\epsilon}_{+} \gamma^{a} \psi_{\mu-}+\frac{1}{2} \bar{\epsilon}_{-} \gamma^{a} \psi_{\mu+}, \quad \delta_{\mathrm{pr}} \psi_{\mu+}=D_{\mu} \epsilon_{+}, \\
\delta_{\mathrm{pr}} \omega_{\mu}{ }^{a b} & =0, \quad \delta_{\mathrm{pr}} \psi_{\mu-}=D_{\mu} \epsilon_{-}+\frac{1}{2} \omega_{\mu}{ }^{a} \gamma_{a 0} \epsilon_{+}
\end{aligned}
$$

The variation of $\omega_{\mu}{ }^{a b}$ is only zero on shell, i.e. upon using the equations of motion of the background fields. The explicit form of these equations of motion are given in Ref. [47]. They also follow by taking the $R \rightarrow \infty$ limit of the formulas in Appendix C. In the same manner we can write the variation of $\omega_{\mu}{ }^{a}$ as

$$
\begin{aligned}
\delta_{\mathrm{pr}} \omega_{\mu}{ }^{a}= & \frac{1}{2} \bar{\epsilon}_{-} \gamma^{0} \hat{\psi}_{\mu}{ }^{a}-+\frac{1}{2} \tau_{\mu} \bar{\epsilon}_{-} \gamma^{0} \hat{\psi}_{0}{ }^{a}{ }_{-}+\frac{1}{4} e_{\mu}{ }^{b} \bar{\epsilon}_{+} \gamma^{b} \hat{\psi}^{a}{ }_{0-} \\
& +\frac{1}{4} \bar{\epsilon}_{+} \gamma^{a} \hat{\psi}_{\mu 0-}
\end{aligned}
$$

where $\hat{\psi}_{\mu \nu-}$ is the covariant curvature of $\psi_{\mu-}$; see Ref. [47] or Appendix $\mathrm{C}$ with $R \rightarrow \infty$. One may check that, to lowest order in fermions, the action (3.20) is invariant under the transformations (3.23), (3.25) and (3.26), upon use of the equations of motion of the background fields.

As a consistency check we have verified that by imposing the gauge-fixing conditions ${ }^{11}$ of Ref. [47] the action and

\footnotetext{
${ }^{11}$ For the bosonic case these conditions are given in Table II.
} 
transformation rules of the $\mathrm{NC}$ superparticle reduce to those of the curved Galilean superparticle.

\section{ADDING A COSMOLOGICAL CONSTANT}

In this section we are going to describe the superparticle in the presence of a cosmological constant. Like in the bosonic case we can derive the action by taking the nonrelativistic limit of a superparticle in AdS space; see e.g. Ref. [16] for an example in ten dimensions.

In the presence of a cosmological constant the relativistic AdS superalgebra in three dimensions is not unique. Instead, in the case of $\mathcal{N}$ supersymmetries, one always finds $\mathcal{N}$ different versions, often referred to as $(p, q) \operatorname{AdS}$ superalgebras [55]. In Appendix A we give both the $(1,1)$ and $(2,0) \mathcal{N}=2$ AdS superalgebras. As explained in Appendix A, the Newton-Hooke superalgebra that we will use below is obtained by contracting the $\mathcal{N}=(2,0)$ AdS superalgebra.

\section{A. Newton-Hooke superparticle}

We find that the action of the $\mathrm{NH}$ superparticle takes the form

$S=\int d \tau \frac{m}{2}\left[\frac{\dot{x}^{i} \dot{x}^{i}}{\dot{t}}-\bar{\theta}_{-} \gamma^{0} \dot{\theta}_{-}-\frac{\dot{t} x^{i} x^{i}}{R^{2}}+\frac{3 \dot{t}}{2 R} \bar{\theta}_{-} \theta_{-}\right]$.

A realization of the Newton-Hooke superalgebra, for which the explicit form is given in Eq. (A6), on the embedding coordinates is given by the following bosonic transformation rules

$\delta t=-\zeta, \quad \delta x^{i}=\lambda^{i}{ }_{k} x^{k}-\xi^{i}(t), \quad \delta \theta_{-}=\frac{1}{4} \lambda^{a b} \gamma_{a b} \theta_{-}$,

supplemented with the following fermionic transformations:

$$
\begin{aligned}
\delta t & =0, \quad \delta x^{i}=-\frac{1}{2} \bar{\epsilon}_{+}(t) \gamma^{i} \theta_{-}, \\
\delta \theta_{-} & =\epsilon_{-}(t)-\frac{\dot{x}^{i}}{2 \dot{t}} \gamma_{0 i} \epsilon_{+}(t)+\frac{x^{i}}{2 R} \gamma_{i} \epsilon_{+}(t) .
\end{aligned}
$$

Here, the time dependence of the parameters $\xi^{i}(t)$ and $\epsilon_{ \pm}(t)$ is given by

$$
\begin{aligned}
\xi^{i}(t) & =v^{i} R \sin \frac{t}{R}+a^{i} \cos \frac{t}{R}, \\
\epsilon_{-}(t) & =\exp \left(\frac{3 t}{2 R} \gamma_{0}\right) \epsilon_{-}, \quad \epsilon_{+}(t)=\exp \left(-\frac{t}{2 R} \gamma_{0}\right) \epsilon_{+} .
\end{aligned}
$$

Like in the $\Lambda=0$ case, the $\epsilon_{+}$transformation is realized linearly while the $\epsilon_{-}$transformation, corresponding to a broken supersymmetry, is not. The superparticle thus corresponds to a $1 / 2$ BPS state. We have verified that the transformations (4.2) and (4.3) leave the NH superparticle action (4.1) invariant.

In the formulation we are using both supersymmetries are time dependent, but $\theta_{-}$is invariant under constant time shifts. Alternatively, one can absorb the time dependence of either $\epsilon_{+}$or $\epsilon_{-}$, but not both at the same time, in a redefinition of $\theta_{-}$. Such a redefinition would introduce a nonvanishing transformation $\delta_{\zeta} \tilde{\theta}_{-}$and shift the $\bar{\theta}_{-} \theta_{-}$term in the action. We prefer to keep the time-dependent description of Eqs. (4.1)-(4.4).

\section{B. Curved Newton-Hooke superparticle}

The curved Newton-Hooke superparticle is obtained in analogy to the curved Galilean superparticle; see Sec. III B. This means that we gauge the spatial translations and one of the supersymmetries, the broken one, such that their parameters become arbitrary time-dependent functions. This introduces the Newton potential $\phi$ and its supersymmetric partner $\psi$. However, based upon our experience in the bosonic case, we expect that either the transformation rules of the NH background fields $\phi$ and $\psi$ differ from the Galilean background fields $\Phi$ and $\Psi$ or their equations of motion change; see the comment in footnote 9. Either way, we cannot make use of the results of Ref. [47]. ${ }^{12}$ Instead, we should first derive the transformations rules of NewtonHooke supergravity as a $1 / R$ modification of Galilean supergravity. Once we have obtained the NH supergravity transformation rules we can use Eq. (2.10) to get the transformation rules of the $\mathrm{NH}$ background fields.

\section{Newton-Hooke supergravity}

We find that the following bosonic and fermionic transformation rules provide a realization of the accelerationextended $\mathrm{NH}$ algebra:

$$
\begin{aligned}
\delta_{\mathrm{pr}} \phi= & \zeta \partial_{t} \phi+\xi^{i}(t) \partial_{i} \phi+\partial_{t} \partial_{t} \xi^{i}(t) x^{i} \\
& +\frac{1}{R^{2}} \xi^{i}(t) x^{i}-\lambda^{i}{ }_{j} x^{j} \partial_{i} \phi+\sigma(t), \\
\delta_{\mathrm{pr}} \psi= & \zeta \partial_{t} \psi+\xi^{i}(t) \partial_{i} \psi+\frac{1}{4} \lambda^{a b} \gamma_{a b} \psi-\lambda_{j}{ }_{j} x^{j} \partial_{i} \psi,
\end{aligned}
$$

and

$$
\begin{gathered}
\delta_{\mathrm{pr}} \phi=\bar{\epsilon}_{-}(t) \gamma^{0} \psi+\frac{1}{2} \partial_{t}\left(\bar{\epsilon}_{+, t} \chi\right)-\frac{x^{i}}{2 R} \bar{\epsilon}_{+, t} \gamma_{0 i} \psi, \\
\delta_{\mathrm{pr}} \psi=\partial_{t} \epsilon_{-}(t)-\frac{3}{2 R} \gamma_{0} \epsilon_{-}(t)-\frac{1}{2} \partial_{i} \phi \gamma_{i 0} \epsilon_{+, t} .
\end{gathered}
$$

\footnotetext{
${ }^{12}$ The equations of motion are needed to close the supersymmetry algebra.
} 
These transformation rules constitute the NH supergravity extension of the Galilean supergravity result given in Ref. [47]. The subscript $t$ on $\epsilon_{+, t}$ indicates that it is a function of time. However, unlike $\xi^{i}(t)$ or $\epsilon_{-}(t)$, the time dependence of $\epsilon_{+, t}$ is a very specific one, namely the one given in Eq. (4.4). The background fields $\phi$ and $\psi$ obey the same equations of motion as in the Galilean case, see Eq. (3.8), and are related to their dual potentials $\Xi$ and $\chi$ by Eqs. (3.10) and (3.9), respectively.

\section{Superparticle action}

Now that we have constructed the Newton-Hooke supergravity theory, it is easy to construct an action for the curved $\mathrm{NH}$ superparticle where the NH supergravity fields occur as background fields. We find that this action is given by

$$
\begin{aligned}
S= & \int d \tau \frac{m}{2}\left[\frac{\dot{x}^{i} \dot{x}^{i}}{\dot{t}}-\bar{\theta}_{-} \gamma^{0} \dot{\theta}_{-}-\frac{\dot{t} x^{i} x^{i}}{R^{2}}+\frac{3 \dot{t}}{2 R} \bar{\theta}_{-} \theta_{-}\right. \\
& \left.-2 \dot{t} \phi+2 \dot{t} \bar{\theta}_{-} \gamma^{0} \psi\right] .
\end{aligned}
$$

The transformation rules of the $\mathrm{NH}$ background fields follow from the $\mathrm{NH}$ supergravity rules given in Eqs. (4.5)-(4.7) after applying the relation (2.10).

As far as we know, for $\Lambda \neq 0$ there is no redefinition akin to (2.23) that would enable us to deduce the transformation rules of the curved Newton-Hooke background fields $\phi$ and $\psi$ from the curved Galilean ones given in Eqs. (3.13) and (3.14), respectively.

\section{Newton-Cartan Newton-Hooke superparticle}

To obtain the NC NH superparticle action we first need to derive the transformation rules of $\mathrm{NC} \mathrm{NH}$ supergravity, viewed as a $1 / R$ modification of the NC supergravity theory constructed in Ref. [47]. We find the following result.

\section{Newton-Cartan Newton-Hooke supergravity}

The bosonic transformation rules of the background fields are given by (3.24) and (2.25). With respect to supersymmetry, the only $1 / R$ modifications occur in the transformation rules of $m_{\mu}, \psi_{\mu+}$ and $\psi_{\mu-}$ :

$$
\begin{gathered}
\delta_{\mathrm{pr}} m_{\mu}=\bar{\epsilon}_{-} \gamma^{0} \psi_{\mu-}-\frac{1}{2} \bar{\epsilon}_{+} \gamma^{a} \theta_{-} e^{\rho}{ }_{a} \partial_{\rho} m_{\mu}-\frac{\left(x^{\nu} e_{\nu}{ }^{a}\right)^{2}}{4 R^{2}} \bar{\epsilon}_{+} \gamma^{0} \psi_{\mu+} \\
-\tau_{\mu} \frac{x^{\nu} x^{\rho} e_{\nu}{ }^{a}}{2 R^{2}}\left(\bar{\epsilon}_{-} \gamma^{a} \psi_{\rho+}+\bar{\epsilon}_{+} \gamma^{a} \psi_{\rho-}\right) \\
\delta_{\mathrm{pr}} \psi_{\mu+}=D_{\mu} \epsilon_{+}+\frac{1}{2 R} \tau_{\mu} \gamma_{0} \epsilon_{+}, \\
\delta_{\mathrm{pr}} \psi_{\mu-}=D_{\mu} \epsilon_{-}+\frac{1}{2} \omega_{\mu}{ }^{a} \gamma_{a 0} \epsilon_{+}-\frac{3}{2 R} \tau_{\mu} \gamma_{0} \epsilon_{-}-\frac{1}{2 R} e_{\mu}{ }^{a} \gamma_{a} \epsilon_{+} .
\end{gathered}
$$

The supersymmetry rules of $\tau_{\mu}, e_{\mu}{ }^{a}$ are undeformed and coincide with the ones of NC supergravity; see (3.25). The above modifications induce the following $1 / R$ modifications in the transformation rules of the dependent fields $\omega_{\mu}^{a b}$ and $\omega_{\mu}{ }^{a}$ :

$$
\delta_{\mathrm{pr}} \omega_{\mu}^{a b}=-\frac{\varepsilon_{a b}}{2 R} \bar{\epsilon}_{+} \gamma^{0} \psi_{\mu+},
$$

$$
\begin{aligned}
\delta_{\mathrm{pr}} \omega_{\mu}{ }^{a}= & \frac{1}{2} \bar{\epsilon}_{-} \gamma^{0} \hat{\psi}_{\mu}{ }^{a}-+\frac{1}{2} \tau_{\mu} \bar{\epsilon}_{-} \gamma^{0} \hat{\psi}_{0}{ }^{a}{ }_{-}+\frac{1}{4} e_{\mu}{ }^{b} \bar{\epsilon}_{+} \gamma^{b} \hat{\psi}^{a}{ }_{0-} \\
& +\frac{1}{4} \bar{\epsilon}_{+} \gamma^{a} \hat{\psi}_{\mu 0-}+\frac{1}{2 R} \bar{\epsilon}_{-} \gamma^{a 0} \psi_{\mu+}+\frac{1}{2 R} \bar{\epsilon}_{+} \gamma^{a 0} \psi_{\mu-} .
\end{aligned}
$$

\section{Superparticle action}

Having constructed the NC NH supergravity theory, it is straightforward to construct the action for the $\mathrm{NC} \mathrm{NH}$ superparticle. We find that the action, up to quartic fermions, is given by

$$
\begin{aligned}
S= & \int d \tau \frac{m}{2}\left[\frac{\dot{x}^{\mu} e_{\mu}{ }^{a} \dot{x}^{\nu} e_{\nu a}}{\dot{x}^{\rho} \tau_{\rho}}-2 m_{\mu} \dot{x}^{\mu}-\bar{\theta}_{-} \gamma^{0} D_{\tau} \theta_{-}\right. \\
& +2 \bar{\theta}_{-} \gamma^{0} \psi_{\mu-} \dot{x}^{\mu}-\frac{\dot{x}^{\mu} e_{\mu a}}{\dot{x}^{\rho} \tau_{\rho}} \bar{\theta}_{-} \gamma^{a} \psi_{\nu+} \dot{x}^{\nu} \\
& \left.-\dot{x}^{\rho} \tau_{\rho} \frac{\left(x^{\mu} e_{\mu}{ }^{a}\right)^{2}}{R^{2}}+\frac{3}{2 R} \dot{x}^{\rho} \tau_{\rho} \bar{\theta}_{-} \theta_{-}\right] .
\end{aligned}
$$

One can show that, upon using the equations of motion of the background fields, see Appendix C, this action is indeed invariant up to quartic fermions. This concludes our description of the NC NH superparticle action.

\section{V. $\kappa$-SYMMETRY}

It is well known that the relativistic superparticle is invariant under an additional infinitely reducible fermionic symmetry called $\kappa$-symmetry $[27,28]$. In the case of strings and branes, this $\kappa$-symmetry is needed to obtain the correct counting of degrees of freedom. It is known from the work of Ref. [15] that in the nonrelativistic case the $\kappa$-symmetry is just a Stückelberg symmetry that acts as a shift on one of the fermionic coordinates. It can easily be gauge fixed upon setting that specific fermionic coordinate equal to zero. Nevertheless, to obtain an elegant superspace description of the nonrelativistic superparticle it might be advantageous to retain this extra fermionic coordinate since it plays the role of one of the superspace coordinates. For this reason we give here the results with $\kappa$-symmetry in two simple cases, namely the Galilean and the NH superparticles. The action and transformation rules of both superparticles are derived in Appendix B by applying the technique of nonlinear realizations. Below we just give 
the results thereby focusing our attention on the $\kappa$-symmetry aspects. For the full details we refer the reader to Appendix B.

\section{A. $\kappa$-symmetric Galilean superparticle}

The $\kappa$-symmetric version of the flat Galilean superparticle action (3.3) depends on an additional fermionic coordinate $\theta_{+}$. The action is given by

$$
S=\int d \tau \frac{m}{2}\left[\frac{\pi^{i} \pi^{i}}{\pi^{0}}-\bar{\theta}_{-} \gamma^{0} \dot{\theta}_{-}\right],
$$

where the line elements $\pi^{0}$ and $\pi^{i}$ are defined as

$$
\pi^{0}=\dot{t}+\frac{1}{4} \bar{\theta}_{+} \gamma^{0} \dot{\theta}_{+}, \quad \pi^{i}=\dot{x}^{i}+\frac{1}{4} \bar{\theta}_{-} \gamma^{i} \dot{\theta}_{+}+\frac{1}{4} \bar{\theta}_{+} \gamma^{i} \dot{\theta}_{-} .
$$

The $\kappa$ transformations that leave the action (5.1) invariant are given by

$$
\begin{aligned}
\delta_{\kappa} t & =\frac{1}{4} \bar{\kappa} \gamma^{0} \theta_{+}, \quad \delta_{\kappa} x^{i}=\frac{1}{4} \bar{\kappa} \gamma^{i} \theta_{-}-\frac{\pi^{k}}{8 \pi^{0}} \bar{\kappa} \gamma_{k} \gamma^{0} \gamma^{i} \theta_{+}, \\
\delta_{\kappa} \theta_{+} & =\kappa, \quad \delta_{\kappa} \theta_{-}=-\frac{\pi^{i}}{2 \pi^{0}} \gamma^{0} \gamma_{i} \kappa .
\end{aligned}
$$

The commutator algebra of all symmetry transformations including local $\kappa$ transformations and worldline reparameterizations closes on shell.

We see from Eq. (5.3) that the $\kappa$ transformation acts as a simple Stückelberg shift symmetry on the embedding coordinate $\theta_{+}$. Therefore, one could just fix this symmetry by imposing the gauge condition

$$
\theta_{+}=0
$$

which leads us back to the formulas of the Galilean superparticle used in Sec. III A.

\section{B. $\kappa$-symmetric Newton-Hooke superparticle}

An analogous construction leads to the $\kappa$-symmetric Newton-Hooke superparticle. We find the following action:

$$
\begin{aligned}
S= & \int d \tau \frac{m}{2}\left[\frac{\pi^{i} \pi^{i}}{\pi^{0}}-\bar{\theta}_{-} \gamma^{0} \dot{\theta}_{-}-\dot{t} \frac{x^{i} x^{i}}{R^{2}}+\frac{3 \dot{t}}{2 R} \bar{\theta}_{-} \theta_{-}\right. \\
& \left.+\frac{\dot{t} x^{i}}{2 R^{2}} \bar{\theta}_{+} \gamma^{i} \theta_{-}-\frac{\dot{t}}{16 R^{2}} \bar{\theta}_{+} \theta_{+} \bar{\theta}_{-} \theta_{-}\right],
\end{aligned}
$$

with the line-elements given by

$$
\pi^{0}=\dot{t}\left(1+\frac{1}{8 R} \bar{\theta}_{+} \theta_{+}\right)+\frac{1}{4} \bar{\theta}_{+} \gamma^{0} \dot{\theta}_{+},
$$

$$
\begin{aligned}
\pi^{i}= & \left(\dot{x}^{i}+\frac{1}{4} \bar{\theta}_{+} \gamma^{i} \dot{\theta}_{-}+\frac{1}{4} \bar{\theta}_{-} \gamma^{i} \dot{\theta}_{+}\right)\left(1-\frac{1}{8 R} \bar{\theta}_{+} \theta_{+}\right) \\
& -\frac{\dot{t}}{4 R}\left(3 \bar{\theta}_{+} \gamma^{0 i} \theta_{-}-\frac{x^{k} \varepsilon_{k i}}{2 R} \bar{\theta}_{+} \theta_{+}\right) .
\end{aligned}
$$

The $\kappa$ transformations read as follows:

$$
\begin{aligned}
\delta t & =\frac{1}{4} \bar{\kappa} \gamma^{0} \theta_{+}, \quad \delta x^{i}=\frac{1}{4} \bar{\kappa} \gamma^{i} \theta_{-}-\frac{\pi^{j}}{8 \pi^{0}} \bar{\kappa} \gamma_{j} \gamma_{i 0} \theta_{+}, \\
\delta \theta_{+} & =\kappa\left(1-\frac{1}{16 R} \bar{\theta}_{+} \theta_{+}\right), \\
\delta \theta_{-} & =-\frac{\pi^{i}}{2 \pi^{0}} \gamma_{i 0} \kappa-\frac{3}{8 R} \gamma_{0} \theta_{-} \bar{\theta}_{+} \gamma^{0} \kappa+\frac{x^{i}}{16 R^{2}} \gamma^{i} \kappa \bar{\theta}_{+} \theta_{+} .
\end{aligned}
$$

Like in the case of the $\kappa$-symmetric Galilean superparticle discussed above the $\kappa$-symmetry can be gauge fixed by imposing the gauge condition $\theta_{+}=0$.

\section{DISCUSSION}

In this paper we have constructed the superparticle actions describing the dynamics of a supersymmetric particle in a 3D curved Galilean and Newton-Cartan supergravity background. Furthermore, we constructed the actions for a superparticle moving in the cosmological extension of these backgrounds by including a cosmological constant. Due to the computational complexity we gave the action in the Newton-Cartan case only up to terms quartic in the fermions. The Newton-Cartan background is characterized by more fields and corresponds to more symmetries than the Galilean background. One can switch between the two backgrounds either by a partial gauging of symmetries (from Galilean to Newton-Cartan) or by gauge fixing some of the symmetries (from Newton-Cartan to Galilean). An important role in the construction is played by symmetries. At several occasions we stressed that, as far as the background fields are concerned, one should use the background transformations and not the proper transformations. The latter are used in the definition of the supergravity multiplet. The relation between the two kind of transformations is given in Eq. (2.10).

A noteworthy feature is that the proof of invariance of the superparticle action requires that the background fields satisfy their equations of motion. This is reminiscent to what happens with the fermionic $\kappa$-symmetry in the relativistic case. We showed in two particular cases that the nonrelativistic superparticle also allows a $\kappa$-symmetric formulation but that in the nonrelativistic case the $\kappa$-symmetry is of a simple Stuickelberg type [15]. Although it is rather trivial, we expect that the formulation with $\kappa$-symmetry is indispensable for a reformulation of our results in terms of a nonrelativistic superspace and superfields; see e.g. Ref. [56]. Such a superspace formulation 
would be useful to construct the superparticle actions in the Newton-Cartan background to all orders in the fermions.

There are several interesting directions in which one could extend our results. First of all, our efforts in this paper were limited to the three-dimensional case. Clearly, it would be desirable to construct the four-dimensional analog of our results. In order to do this, one should first be able to construct the Galilean and Newton-Cartan supergravity multiplets in four spacetime dimensions. So far, this has not yet been achieved. Another generalization of our results would be to go from superparticles to superstrings or even super $p$-branes. This would require taking a "stringy" generalization of the nonrelativistic limits we have been considering here; see e.g. Ref. [48]. The case of a nonrelativistic superstring in a flat background was already considered in Ref. [16]. In the case of a nonrelativistic curved background one could apply holography and study the corresponding nonrelativistic supersymmetric boundary theory.

In the case of a (super)particle propagating in three spacetime dimensions the Galilei algebra contains a second central charge that could be included as well. This leads to the notion of a noncommutative nonrelativistic (super) particle where the embedding coordinates are noncommutative with respect to the Dirac brackets [57,58]. Finally, we have found that some of our superparticles are 1/2 BPS, corroborating recent results for relativistic superparticles [59]. It would be interesting to verify whether the statement that "all superparticles are BPS" [59] applies to nonrelativistic superparticles as well.

\section{ACKNOWLEDGMENTS}

We thank Jelle Hartong and Kiyoshi Kamimura for inspiring discussions and comments on the draft. E. B. and J.R. would like to thank the organizers of the Simons Summer Workshop 2013 on Mathematics and Physics for its hospitality and generous financial support. J. G. acknowledges the hospitality at the Department of Theoretical Physics of the University of Groningen where part of this work was done. J. G. also acknowledges partial financial support from the Dutch research organization FOM and from FPA 2010-20807, 2009 SGR502, CPAN, and Consolider CSD 2007-0042. The work of M. K. is supported by the Ubbo Emmius Programme administered by the Graduate School of Science, University of Groningen. L. P. acknowledges support by the Consejo Nacional de Ciencia y Tecnología (CONACyT), the Universidad Nacional Autónoma de México via the project UNAM-PAPIIT IN109013 and an Ubbo Emmius sandwich scholarship from the University of Groningen. The work of J.R. was supported by the START project Y 435-N16 of the Austrian Science Fund (FWF). T.Z. acknowledges support by a grant of the Dutch Academy of Sciences (KNAW).

\section{APPENDIX A: NEWTON-HOOKE SUPERALGEBRA}

The NH (super)algebra can be derived as a contraction of the AdS (super)algebra. In the case of two supersymmetries there are two independent versions of the latter one, the socalled $\mathcal{N}=(1,1)$ and $\mathcal{N}=(2,0)$ algebras. In the main text we use only the $\mathcal{N}=(2,0)$ algebra for reasons we will explain below.

We proceed by discussing the contraction of the 3D $\mathcal{N}=(2,0)$ AdS algebra. The basic commutators are given by $(A=0,1,2)$

$$
\begin{aligned}
{\left[M_{A B}, M_{C D}\right] } & =2 \eta_{A[C} M_{D] B}-2 \eta_{B[C} M_{D] A}, \\
{\left[M_{A B}, Q^{i}\right] } & =-\frac{1}{2} \gamma_{A B} Q^{i}, \\
{\left[M_{A B}, P_{C}\right] } & =-2 \eta_{C[A} P_{B]}, \quad\left[P_{A}, Q^{i}\right]=x \gamma_{A} Q^{i}, \\
{\left[P_{A}, P_{B}\right]=} & 4 x^{2} M_{A B}, \quad\left[\mathcal{R}, Q^{i}\right]=2 x \varepsilon^{i j} Q^{j}, \\
\left\{Q_{\alpha}^{i}, Q_{\beta}^{j}\right\}= & 2\left[\gamma^{A} C^{-1}\right]_{\alpha \beta} P_{A} \delta^{i j}+2 x\left[\gamma^{A B} C^{-1}\right]_{\alpha \beta} M_{A B} \delta^{i j} \\
& +2 C_{\alpha \beta}^{-1} \varepsilon^{i j} \mathcal{R} .
\end{aligned}
$$

Here, $P_{A}, M_{A B}, \mathcal{R}$ and $Q_{\alpha}^{i}$ are the generators of spacetime translations, Lorentz rotations, $\mathrm{SO}(2)$ R-symmetry transformations and supersymmetry transformations, respectively. The bosonic generators $P_{A}, M_{A B}$ and $\mathcal{R}$ are anti-Hermitian while the fermionic generators $Q_{\alpha}^{i}$ are Hermitian. The parameter $x$ is a contraction parameter. Note that the generator of the $\mathrm{SO}(2) \mathrm{R}$-symmetry becomes the central element of the Poincare algebra in the flat limit $x \rightarrow 0$.

To show that the above algebra corresponds to the $\mathcal{N}=(2,0)$ AdS algebra it is convenient to define the new generators

$$
M_{C}=\epsilon_{C A B} M^{A B}, \quad J_{A}^{ \pm}=P_{A} \pm x M_{A} .
$$

In terms of these new generators we obtain the following (anti)commutation relations:

$\left[J_{A}^{+}, Q^{i}\right]=2 x \gamma_{A} Q^{i}, \quad\left\{Q_{\alpha}^{i}, Q_{\beta}^{j}\right\}=2\left[\gamma^{A} C^{-1}\right]_{\alpha \beta} J_{A}^{+} \delta^{i j}$,

while the charges $Q^{i}$ do not transform under $J_{A}^{-}$. This identifies the algebra as the $\mathcal{N}=(2,0)$ AdS algebra.

To make the nonrelativistic contraction we define new supersymmetry charges by

$$
Q_{\alpha}^{ \pm}=\frac{1}{2}\left(Q_{\alpha}^{1} \pm \gamma_{0} Q_{\alpha}^{2}\right)
$$

and rescale the generators with a parameter $\omega$ as follows: 


$$
\begin{aligned}
& P_{0}=\omega Z+\frac{1}{2 \omega} H, \quad \mathcal{R}=-\omega Z+\frac{1}{2 \omega} H, \quad M_{a 0}=\omega G_{a}, \\
& Q^{+}=\frac{1}{\sqrt{\omega}} \tilde{Q}^{+}, \quad Q^{-}=\sqrt{\omega} \tilde{Q}^{-} .
\end{aligned}
$$

We also set $x=1 /(2 \omega R)$. Taking the limit $\omega \rightarrow \infty$ and dropping the tildes on the $Q^{ \pm}$we get the following 3D $\mathcal{N}=(2,0)$ Newton-Hooke superalgebra:

$$
\begin{aligned}
{\left[J_{a b},(P / G)_{c}\right] } & =-2 \delta_{c[a}(P / G)_{b]}, \quad\left[H, G_{a}\right]=P_{a}, \\
{\left[H, P_{a}\right] } & =-\frac{1}{R^{2}} G_{a}, \quad\left[J_{a b}, Q^{ \pm}\right]=-\frac{1}{2} \gamma_{a b} Q^{ \pm}, \\
{\left[H, Q^{+}\right] } & =-\frac{1}{2 R} \gamma_{0} Q^{+}, \quad\left[H, Q^{-}\right]=\frac{3}{2 R} \gamma_{0} Q^{-}, \\
{\left[G_{a}, Q^{+}\right] } & =-\frac{1}{2} \gamma_{a 0} Q^{-}, \quad\left[P_{a}, Q^{+}\right]=\frac{1}{2 R} \gamma_{a} Q^{-}, \\
\left\{Q_{\alpha}^{+}, Q_{\beta}^{+}\right\} & =\left[\gamma^{0} C^{-1}\right]_{\alpha \beta} H+\frac{1}{2 R}\left[\gamma^{a b} C^{-1}\right]_{\alpha \beta} J_{a b}, \\
\left\{Q_{\alpha}^{+}, Q_{\beta}^{-}\right\} & =\left[\gamma^{a} C^{-1}\right]_{\alpha \beta} P_{a}+\frac{1}{R}\left[\gamma^{a 0} C^{-1}\right]_{\alpha \beta} G_{a} .
\end{aligned}
$$

The central extension $Z$ that leads to the Bargmann version of the $\mathrm{NH}$ superalgebra occurs in the following (anti) commutation relations:

$\left[P_{a}, G_{b}\right]=\delta_{a b} Z, \quad\left\{Q_{\alpha}^{-}, Q_{\beta}^{-}\right\}=2\left[\gamma^{0} C^{-1}\right]_{\alpha \beta} Z$.

The reason why we do not use the $\mathcal{N}=(1,1)$ AdS algebra for the contraction is essentially the same reason as why we are interested in $\mathcal{N}=2$ rather than $\mathcal{N}=1$ algebras. The authors of Ref. [47] gauged the $\mathcal{N}=2$ Galilei superalgebra in order to obtain commutator relations that yield $\{Q, Q\} \sim H$ and $\{Q, Q\} \sim P$, that is, the commutator of two supersymmetries gives time and space translations. For $\mathcal{N}=1$ one can have only one of them. The $\mathcal{N}=(1,1)$ AdS algebra is equal to the direct product $\mathrm{OSp}(1 \mid 2) \otimes O S p(1 \mid 2)$. Taking the nonrelativistic contraction thereof amounts to taking the simultaneous contractions of two independent $\mathcal{N}=1$ algebras. However, we already argued that this cannot lead to a superalgebra of the desired form.

\section{APPENDIX B: NONLINEAR REALIZATIONS}

In this appendix we obtain the action and transformation rules for the flat Galilean and Newton-Hooke superparticles by using the method of nonlinear realizations $[53,54] .{ }^{13}$ We will derive in the first subsection the $\kappa$ symmetric action and transformation rules of the Galilean superparticle; see e.g. Refs. [62,63]. In the second

\footnotetext{
${ }^{13}$ For an early application of this method in a different situation than the one considered in this work, namely to the construction of worldline actions of conformal and superconformal particles, see Refs. [60,61].
}

subsection we will do the same for the NH superparticle. The normalizations of the (to-be) embedding coordinates that occur in this appendix differ from those in the main text.

\section{1. $\kappa$-symmetric Galilean superparticle}

The starting point is the $\mathcal{N}=2$ Bargmann superalgebra given in Sec. III. We derive the transformation rules for the coordinates $\left(t, x^{i}, s, \theta_{-}^{\alpha}, \theta_{+}^{\alpha}, k^{i}\right)$ using the coset

$$
g=e^{H t} e^{P_{i} x^{i}} e^{Z s} e^{Q_{\alpha}^{-} \theta_{-}^{\alpha}} e^{Q_{\alpha}^{+} \theta_{+}^{\alpha}} e^{G_{i} k^{i}} .
$$

This leads to the Maurer-Cartan form

$$
\Omega=g^{-1} d g=H L_{H}+\cdots-\bar{Q}^{-} L_{-}-\bar{Q}^{+} L_{+},
$$

with the $L$ 's given by

$$
\begin{aligned}
L_{H}= & d t-\frac{1}{2} \bar{\theta}_{+} \gamma^{0} d \theta_{+}, \\
L_{Z}= & d s-\bar{\theta}_{-} \gamma^{0} d \theta_{-}+\frac{k^{i} k^{i}}{2}\left(d t-\frac{1}{2} \bar{\theta}_{+} \gamma^{0} d \theta_{+}\right) \\
& +\left(d x^{i}-\bar{\theta}_{+} \gamma^{i} d \theta_{-}\right) k^{i}, \\
L_{P}^{i}= & d x^{i}-\bar{\theta}_{+} \gamma^{i} d \theta_{-}+k^{i}\left(d t-\frac{1}{2} \bar{\theta}_{+} \gamma^{0} d \theta_{+}\right), \quad L_{G}^{i}=d k^{i}, \\
L_{J}^{a b}= & 0, \quad L_{-}=d \theta_{-}-\frac{1}{2} \gamma_{i 0} d \theta_{+} k^{i}, \quad L_{+}=d \theta_{+} .
\end{aligned}
$$

It is convenient to define the line elements

$$
\pi^{0}=\dot{t}-\frac{1}{2} \bar{\theta}_{+} \gamma^{0} \dot{\theta}_{+}, \quad \pi^{i}=\dot{x}^{i}-\bar{\theta}_{+} \gamma^{i} \dot{\theta}_{-},
$$

which are related to the Maurer-Cartan form via the pullbacks

$$
\left(L_{H}\right)^{*}=\pi^{0}, \quad\left(L_{P}\right)^{*}=\pi^{i}+k^{i} \pi^{0} .
$$

The action of the Galilean superparticle is given by the pullback of all $L$ 's that are invariant under rotations, hence

$$
\begin{aligned}
S & =a \int\left(L_{H}\right)^{*}+\int\left(L_{Z}\right)^{*} \\
& =\int d \tau\left[-\frac{a}{2} \bar{\theta}_{+} \gamma^{0} \dot{\theta}_{+}-\bar{\theta}_{-} \gamma^{0} \dot{\theta}_{-}-\frac{\pi^{i} \pi^{i}}{2 \pi^{0}}\right] .
\end{aligned}
$$

Here, we replaced the Goldstone field $k^{i}$ by its equation of motion $k^{i}=-\pi^{i} / \pi^{0}$. This procedure is know as inverse Higgs mechanism [64]; see also Ref. [65]. The bosonic transformations of the embedding coordinates are 


$$
\begin{gathered}
\delta t=-\zeta, \quad \delta x^{i}=\lambda^{i}{ }_{k} x^{k}-a^{i}+v^{i} t+\frac{v^{k} \varepsilon_{k i}}{4} \bar{\theta}_{+} \theta_{+}, \\
\delta \theta_{+}=\frac{1}{4} \lambda^{a b} \gamma_{a b} \theta_{+}, \quad \delta \theta_{-}=\frac{1}{4} \lambda^{a b} \gamma_{a b} \theta_{-}+\frac{v^{i}}{2} \gamma^{i 0} \theta_{+},
\end{gathered}
$$

and the supersymmetry transformations are

$\delta t=\frac{1}{2} \bar{\epsilon}_{+} \gamma^{0} \theta_{+}, \quad \delta x^{i}=\bar{\epsilon}_{+} \gamma^{i} \theta_{-}, \quad \delta \theta_{ \pm}=\epsilon_{ \pm}$.

These transformations leave the action (B6) and all $L$ 's, in particular the line elements (B4), invariant.

To derive an action that is invariant under $\kappa$ transformations we need to find a fermionic gauge transformation that leaves $L_{H}$ and/or $L_{Z}$ invariant. ${ }^{14}$ The variation of $L_{H}$ and $L_{Z}$ under gauge transformations is given by

$$
\begin{gathered}
\delta L_{H}=d\left[\delta z_{H}\right]-\bar{L}_{+} \gamma^{0}\left[\delta z_{+}\right] \\
\delta L_{Z}=d\left[\delta z_{Z}\right]-2 \bar{L}_{-} \gamma^{0}\left[\delta z_{-}\right]-\delta_{a b}\left(L_{P}^{a}\left[\delta z_{G}^{b}\right]-L_{G}^{a}\left[\delta z_{P}^{b}\right]\right) .
\end{gathered}
$$

For $\kappa$ transformations we find, using the explicit expressions for $L_{+}$and $L_{-}$,

$$
\begin{gathered}
\left(\delta L_{H}\right)^{*}=\left[\delta \bar{z}_{+}\right] \gamma^{0} \dot{\theta}_{+}, \\
\left(\delta L_{Z}\right)^{*}=2\left[\delta \bar{z}_{-}\right] \gamma^{0}\left(\dot{\theta}_{-}-\frac{1}{2} \gamma_{i 0} \dot{\theta}_{+} k^{i}\right) .
\end{gathered}
$$

It follows that to obtain a $\kappa$-symmetric action we need to take the pullback of either $L_{H}$ or $L_{Z}$, with $\left[\delta z_{+}\right]=0$ or $\left[\delta z_{-}\right]=0$, respectively. We focus here on the second case; i.e. we choose $a=0$. Then the action and $\kappa$-symmetry rules are given by

$$
S=\int\left(L_{Z}\right)^{*}, \quad\left[\delta z_{+}\right]=\kappa, \quad\left[\delta z_{-}\right]=0,
$$

where $\kappa$ is an arbitrary (local) parameter. Using this we find the following $\kappa$ transformations of the coordinates:

$$
\begin{aligned}
\delta t & =-\frac{1}{2} \bar{\kappa} \gamma^{0} \theta_{+}, \quad \delta x^{i}=-\frac{\pi^{j}}{2 \pi^{0}} \bar{\theta}_{+} \gamma^{i} \gamma_{j 0} \kappa, \\
\delta \theta_{+} & =\kappa, \quad \delta \theta_{-}=-\frac{\pi^{i}}{2 \pi^{0}} \gamma_{i 0} \kappa .
\end{aligned}
$$

The corresponding $\kappa$-symmetric action is given by

\footnotetext{
${ }^{14}$ The case of the $S U(1,1 \mid 2)$ superconformal particle is discussed in Ref. [66].
}

$$
S=\int d \tau \frac{m}{2}\left[-2 \bar{\theta}_{-} \gamma^{0} \dot{\theta}_{-}-\frac{\pi^{i} \pi^{i}}{\pi^{0}}\right] .
$$

To compare to the action and transformations rules given in the main text one needs to make the following redefinitions,

$$
\begin{aligned}
t & \rightarrow-t, \quad x^{i} \rightarrow-x^{i}+\frac{1}{2} \bar{\theta}_{+} \gamma^{i} \theta_{-}, \\
\pi^{0} & \rightarrow-\pi^{0}, \quad \pi^{i} \rightarrow-\pi^{i},
\end{aligned}
$$

and rescale all spinors by $1 / \sqrt{2}$.

\section{2. $\boldsymbol{\kappa}$-symmetric Newton-Hooke superparticle}

The previous subsection was a warming up exercise for the derivation of the NH superparticle which is a deformation of the Galilean superparticle. Here, we give only the main results. Starting from the NH superalgebra given in Appendix A we choose the same coset as in the previous subsection, see Eq. (B1), and find the following expressions for the $L$ 's:

$$
\begin{aligned}
& L_{H}=d t\left(1-\frac{1}{4 R} \bar{\theta}_{+} \theta_{+}\right)-\frac{1}{2} \bar{\theta}_{+} \gamma^{0} d \theta_{+}, \\
& L_{P}^{i}=d x^{i}\left(1+\frac{1}{4 R} \bar{\theta}_{+} \theta_{+}\right)+\frac{d t}{2 R}\left(3 \bar{\theta}_{+} \gamma^{0 i} \theta_{-}-\frac{x^{k} \epsilon_{k i}}{2 R} \bar{\theta}_{+} \theta_{+}\right) \\
& -\bar{\theta}_{+} \gamma^{i} d \theta_{-}+k^{i} L_{H}, \\
& L_{Z}=d s+\frac{d t}{2 R}\left(\frac{x^{i} x^{i}}{R}+3 \bar{\theta}_{-} \theta_{-}\right)-\bar{\theta}_{-} \gamma^{0} d \theta_{-}+k^{i} L_{P}^{i} \\
& -\frac{k^{i} k^{i}}{2} L_{H}, \\
& L_{G}^{i}=\frac{d t}{R}\left(-\frac{x^{i}}{R}-\frac{x^{i}}{4 R^{2}} \bar{\theta}_{+} \theta_{+}+\frac{3}{2 R} \bar{\theta}_{+} \gamma^{i} \theta_{-}\right)-\frac{d x^{k} \epsilon_{k i}}{4 R^{2}} \bar{\theta}_{+} \theta_{+} \\
& -\frac{1}{R} \bar{\theta}_{+} \gamma^{i 0} d \theta_{-}-2 k^{k} L_{J}^{k i}+d k^{i}, \\
& L_{J}^{a b}=-\frac{d t \epsilon_{a b}}{8 R^{2}} \bar{\theta}_{+} \theta_{+}-\frac{1}{4 R} \bar{\theta}_{+} \gamma^{a b} d \theta_{+}, \\
& L_{-}=d \theta_{-}\left(1-\frac{1}{2 R} \bar{\theta}_{+} \theta_{+}\right) \\
& -\frac{d t}{2 R}\left(3 \gamma_{0} \theta_{-}-\frac{3}{2 R} \gamma_{0} \theta_{-} \bar{\theta}_{+} \theta_{+}-\frac{x^{i}}{R} \gamma^{i 0} \theta_{+}\right) \\
& -\frac{d x^{i}}{2 R} \gamma^{i} \theta_{+}-\frac{k^{i}}{2} \gamma_{i 0} L_{+} \\
& L_{+}=d \theta_{+}+\frac{d t}{2 R} \gamma_{0} \theta_{+} \text {. }
\end{aligned}
$$

As before we use $L_{P}^{i}$ and $L_{H}$ to define line elements using the definition (B5). These will be useful when we write the action.

Using the Maurer-Cartan form one can derive the transformation rules of the Goldstone fields that realize 
the $\mathrm{NH}$ superalgebra (A6). We find that the bosonic transformation rules are given by

$$
\begin{aligned}
\delta t= & -\zeta, \\
\delta x^{i}= & \lambda^{i}{ }^{2} x^{k}-a^{i} \cos \frac{t}{R}+\frac{a^{k} \varepsilon_{k i}}{4 R} \sin \frac{t}{R} \bar{\theta}_{+} \theta_{+}+v^{i} R \sin \frac{t}{R} \\
& +\frac{v^{k} \varepsilon_{k i}}{4} \cos \frac{t}{R} \bar{\theta}_{+} \theta_{+}, \\
\delta \theta_{+}= & \frac{1}{4} \lambda^{a b} \gamma_{a b} \theta_{+}, \\
\delta \theta_{-}= & \frac{1}{4} \lambda^{a b} \gamma_{a b} \theta_{-}+\frac{a^{i}}{2 R} \sin \frac{t}{R} \gamma^{i 0} \theta_{+}+\frac{v^{i}}{2} \cos \frac{t}{R} \gamma^{i 0} \theta_{+} .
\end{aligned}
$$

We do not give the transformation rules for $k^{i}$ and $s$ since we do not need them in the following. The transformation rules under the $\epsilon_{-}$-supersymmetry transformations are given by

$$
\delta \theta_{-}=\epsilon_{-}(t)=\exp \left(\frac{3 t}{2 R} \gamma_{0}\right) \epsilon_{-},
$$

while all others fields are invariant (except $s$ ). This transformation leaves the line elements invariant. For the $\epsilon_{+}$ transformations we find the following rules:

$$
\begin{aligned}
\delta t= & \frac{1}{2} \bar{\epsilon}_{+}(t) \gamma^{0} \theta_{+}, \quad \delta x^{i}=\bar{\epsilon}_{+}(t) \gamma^{i} \theta_{-}\left(1-\frac{1}{4 R} \bar{\theta}_{+} \theta_{+}\right), \\
\delta \theta_{+}= & \epsilon_{+}(t)\left(1-\frac{1}{8 R} \bar{\theta}_{+} \theta_{+}\right), \\
\delta \theta_{-}= & \frac{x^{i}}{2 R} \gamma_{i} \epsilon_{+}(t)\left(1+\frac{1}{4 R} \bar{\theta}_{+} \theta_{+}\right) \\
& -\frac{1}{2 R} \gamma_{i} \theta_{+} \bar{\epsilon}_{+}(t) \gamma^{i} \theta_{-}+\frac{3}{4 R} \gamma_{0} \theta_{-} \bar{\epsilon}_{+}(t) \gamma^{0} \theta_{+}, \quad \text { (B20) }
\end{aligned}
$$

with

$$
\epsilon_{+}(t)=\exp \left(\frac{-t}{2 R} \gamma_{0}\right) \epsilon_{+} \cdot
$$

We are now ready to derive the action and $\kappa$-transformation rules. Like in the Galilean case we need to require that the action consists either of the pullback of $L_{H}$ or $L_{Z}$ but not both, together with $\left[\delta z_{+}\right]=0$ or $\left[\delta z_{-}\right]=0$, respectively. We focus again on the second case, i.e. we take

$$
S=\int\left(L_{Z}\right)^{*}, \quad\left[\delta z_{+}\right]=\kappa, \quad\left[\delta z_{-}\right]=0,
$$

with $\kappa$ an arbitrary (local) parameter. Together with the rescalings (B16) and $R \rightarrow-R$ this leads to the action and transformation rules of the $\kappa$-symmetric $\mathrm{NH}$ superparticle given in Sec. V; see Eqs. (5.5) and (5.8). After fixing the $\kappa$-symmetry by imposing the condition $\theta_{+}=0$ these formulas reduce to those of the $\mathrm{NH}$ superparticle given in Sec. IVA.

\section{APPENDIX C: NC NH SUPERPARTICLE: SOME USEFUL FORMULAS}

In this appendix we collect a few formulas that are needed to show that the NC NH superparticle action (4.14) is invariant under the bosonic and fermionic symmetries given in Sec. IV C.

The transformation rules of the background fields of $\mathrm{NC} \mathrm{NH}$ supergravity are given by those of NC supergravity plus the $1 / R$ corrections denoted in Eqs. (4.10) and (4.11). All constraints and equations of motion on the background fields follow from curvatures of those fields. These curvatures can be derived directly from the $\mathrm{NH}$ superalgebra (A6) (with an obvious redefinition of $m_{\mu}$ ):

$$
\begin{aligned}
\hat{R}_{\mu \nu}(H)= & 2 \partial_{[\mu} \tau_{\nu]}-\frac{1}{2} \bar{\psi}_{+[\mu} \gamma^{0} \psi_{\nu]+}, \\
\hat{R}_{\mu \nu}{ }^{a}(P)= & 2 \partial_{[\mu} e_{\nu]}^{a}-2 \omega_{[\mu}{ }^{a b} e_{\nu] b}-2 \omega_{[\mu}{ }^{a} \tau_{\nu]}-\bar{\psi}_{+[\mu} \gamma^{a} \psi_{\nu]-}, \\
\hat{R}_{\mu \nu}{ }^{a}(G)= & 2 \partial_{[\mu} \omega_{\nu]}{ }^{a}-2 \omega_{[\mu}{ }^{a b} \omega_{\nu] b}+\frac{2}{R^{2}} e_{[\mu}{ }^{a} \tau_{\nu]} \\
& -\frac{1}{R} \bar{\psi}_{[\mu-} \gamma^{a 0} \psi_{\nu]+}, \\
\hat{R}_{\mu \nu}{ }^{a b}(J)= & 2 \partial_{[\mu} \omega_{\nu]}{ }^{a b}+\frac{1}{2 R} \bar{\psi}_{\mu+} \gamma^{a b} \psi_{\nu+}, \\
\hat{R}_{\mu \nu}(Z)= & 2 \partial_{[\mu}\left(m_{\nu]}+\tau_{\nu]} \frac{\left(x^{\rho} e_{\rho}{ }^{a}\right)^{2}}{2 R^{2}}\right) \\
& -2 \omega_{[\mu}{ }^{a} e_{\nu] a}-\bar{\psi}_{\mu-} \gamma^{0} \psi_{\nu-}, \\
\hat{\psi}_{\mu \nu+}= & 2 \partial_{[\mu} \psi_{\nu]+}-\frac{1}{2} \omega_{[\mu}{ }^{a b} \gamma_{a b} \psi_{\nu]}+\frac{1}{R} \tau_{[\mu} \gamma_{0} \psi_{\nu]+}, \\
\hat{\psi}_{\mu \nu-}= & 2 \partial_{[\mu} \psi_{\nu]-}-\frac{1}{2} \omega_{[\mu}{ }^{a b} \gamma_{a b} \psi_{\nu]-}+\omega_{[\mu}{ }^{a} \gamma_{a 0} \psi_{\nu]+} \\
& -\frac{3}{R} \tau_{[\mu} \gamma_{0} \psi_{\nu]-}-\frac{1}{R}^{1} e_{[\mu}{ }^{a} \gamma_{a} \psi_{\nu]+} \cdot
\end{aligned}
$$

The curvatures $\hat{R}_{\mu \nu}{ }^{a}(P)$ and $\hat{R}_{\mu \nu}(Z)$ do not change with respect to the NC case discussed in Ref. [47]. Therefore, one can still use these, by setting $\hat{R}_{\mu \nu}{ }^{a}(P)=$ $\hat{R}_{\mu \nu}(Z)=0$, to solve for $\omega_{\mu}{ }^{a b}$ and $\omega_{\mu}{ }^{a}$ in terms of the other fields. Furthermore one can impose the additional constraints

$$
\hat{R}_{\mu \nu}(H)=\hat{\psi}_{\mu \nu+}=\hat{R}_{\mu \nu}^{a b}(J)=0 .
$$

In order to obtain on-shell closure of the supersymmetry algebra on the background fields the curvature $\hat{\psi}_{\mu \nu-}$ needs to obey

$$
\gamma^{\mu} \tau^{\nu} \hat{\psi}_{\mu \nu-}=0, \quad e_{a}^{\mu} e_{b}^{\nu} \hat{\psi}_{\mu \nu-}=0 .
$$

The additional constraints $(\mathrm{C} 2)$ and the equations of motion (C3) are needed to proof invariance of the NC NH superparticle action (4.14). 
[1] Y. Nishida and D. T. Son, Phys. Rev. D 76, 086004 (2007).

[2] D. Son, Phys. Rev. D 78, 046003 (2008).

[3] K. Balasubramanian and J. McGreevy, Phys. Rev. Lett. 101, 061601 (2008).

[4] C. P. Herzog, M. Rangamani, and S. F. Ross, J. High Energy Phys. 11 (2008) 080.

[5] S. Kachru, X. Liu, and M. Mulligan, Phys. Rev. D 78, 106005 (2008).

[6] S. A. Hartnoll, Classical Quantum Gravity 26, 224002 (2009).

[7] A. Bagchi and R. Gopakumar, J. High Energy Phys. 07 (2009) 037.

[8] M. H. Christensen, J. Hartong, N. A. Obers, and B. Rollier, Phys. Rev. D 89, 061901 (2014).

[9] M. H. Christensen, J. Hartong, N. A. Obers, and B. Rollier, J. High Energy Phys. 01 (2014) 057.

[10] B. Julia and H. Nicolai, Nucl. Phys. B439, 291 (1995).

[11] C. Duval and P. A. Horvathy, J. Phys. A 42, 465206 (2009).

[12] D. T. Son, arXiv:1306.0638.

[13] J. Gomis and H. Ooguri, J. Math. Phys. (N.Y.) 42, 3127 (2001).

[14] U. H. Danielsson, A. Guijosa, and M. Kruczenski, J. High Energy Phys. 10 (2000) 020.

[15] J. Gomis, K. Kamimura, and P. K. Townsend, J. High Energy Phys. 11 (2004) 051.

[16] J. Gomis, J. Gomis, and K. Kamimura, J. High Energy Phys. 12 (2005) 024.

[17] D. E. Berenstein, J. M. Maldacena, and H. S. Nastase, J. High Energy Phys. 04 (2002) 013.

[18] R. Casalbuoni, Nuovo Cimento A 33, 389 (1976).

[19] L. Brink and J. Schwarz, Phys. Lett. 100B, 310 (1981).

[20] R. Puzalowski, Acta Phys. Austriaca 50, 45 (1978).

[21] T. Clark and S. Love, Nucl. Phys. B231, 91 (1984).

[22] J. P. Gauntlett, J. Gomis, and P. Townsend, Phys. Lett. B 248, 288 (1990).

[23] J. de Azcarraga and D. Ginestar, J. Math. Phys. (N.Y.) 32, 3500 (1991).

[24] C. Duval and P. Horvathy, J. Math. Phys. (N.Y.) 35, 2516 (1994).

[25] O. Bergman and C. B. Thorn, Phys. Rev. D 52, 5997 (1995).

[26] O. Bergman, Int. J. Mod. Phys. A 12, 1173 (1997).

[27] J. A. de Azcarraga and J. Lukierski, Phys. Lett. 113B, 170 (1982).

[28] W. Siegel, Phys. Lett. 128B, 397 (1983).

[29] G. W. Moore and P. C. Nelson, Phys. Rev. Lett. 53, 1519 (1984).

[30] C. Hull and A. Van Proeyen, Phys. Lett. B 351, 188 (1995).

[31] A. Trautmann, Brandeis Summer Institute, Lectures on General Relativity, Waltham, Massachusets, 1965 (Prentice-Hall, Englewood Cliffs, NJ, 1965).

[32] R. De Pietri, L. Lusanna, and M. Pauri, Classical Quantum Gravity 12, 219 (1995).

[33] R. Banerjee, A. Mitra, and P. Mukherjee, arXiv:1404.4491.

[34] J.-M. Lévy-Leblond, Group Theory and Applications, edited by E. M. Loebl (Academic, New York, 1971), Vol. II, p. 221.
[35] J. Lukierski, P. Stichel, and W. Zakrzewski, Phys. Lett. B 650, 203 (2007).

[36] R. Andringa, E. Bergshoeff, S. Panda, and M. de Roo, Classical Quantum Gravity 28, 105011 (2011).

[37] R. Aldrovandi, A. Barbosa, L. Crispino, and J. Pereira, Classical Quantum Gravity 16, 495 (1999).

[38] H. Bacry and J. Levy-Leblond, J. Math. Phys. (N.Y.) 9, 1605 (1968).

[39] J. Derome and J. Dubois, Nuovo Cimento Soc. Ital. Fis. 9, 351 (1972).

[40] Y.-h. Gao, Advanced Lectures in Mathematics, edited by K. Liu, S.-T. Yau, and C. Zhu, Superstring Theory Vol. I (International Press, Boston, 2002), pp. 271-310.

[41] G. Gibbons and C. Patricot, Classical Quantum Gravity 20, 5225 (2003).

[42] J. Brugues, J. Gomis, and K. Kamimura, Phys. Rev. D 73, 085011 (2006).

[43] M. Sakaguchi and K. Yoshida, J. High Energy Phys. 10 (2006) 078.

[44] J. Lukierski, P. Stichel, and W. Zakrzewski, Eur. Phys. J. C 55, 119 (2008).

[45] J. Gomis and J. Lukierski, Phys. Lett. B 664, 107 (2008).

[46] A. Galajinsky, Phys. Lett. B 680, 510 (2009).

[47] R. Andringa, E. A. Bergshoeff, J. Rosseel, and E. Sezgin, Classical Quantum Gravity 30, 205005 (2013).

[48] R. Andringa, E. Bergshoeff, J. Gomis, and M. de Roo, Classical Quantum Gravity 29, 235020 (2012).

[49] J. Lévy-Leblond, Commun. Math. Phys. 12, 64 (1969).

[50] G. Marmo, G. Morandi, A. Simoni, and E. Sudarshan, Phys. Rev. D 37, 2196 (1988).

[51] C. Duval, Classical Quantum Gravity 10, 2217 (1993).

[52] K. Kuchar, Phys. Rev. D 22, 1285 (1980).

[53] S. R. Coleman, J. Wess, and B. Zumino, Phys. Rev. 177, 2239 (1969).

[54] J. Callan, G. Curtis, S. R. Coleman, J. Wess, and B. Zumino, Phys. Rev. 177, 2247 (1969).

[55] A. Achucarro and P. Townsend, Phys. Lett. B 180, 89 (1986).

[56] J. Lukierski, I. Prochnicka, P. Stichel, and W. Zakrzewski, Phys. Lett. B 639, 389 (2006).

[57] J. Lukierski, P. C. Stichel, and W. J. Zakrzewski, Ann. Phys. (N.Y.) 260, 224 (1997).

[58] C. Batlle, J. Gomis, and K. Kamimura, SIGMA 10, 011 (2014).

[59] L. Mezincescu, A. J. Routh, and P. K. Townsend, J. Phys. A 47, 175401 (2014).

[60] E. Ivanov, S. Krivonos, and V. Leviant, J. Phys. A 22, 345 (1989).

[61] E. Ivanov, S. Krivonos, and V. Leviant, J. Phys. A 22, 4201 (1989).

[62] J. Gomis, K. Kamimura, and P. C. West, Classical Quantum Gravity 23, 7369 (2006).

[63] J. Gomis, K. Kamimura, and P. C. West, J. High Energy Phys. 10 (2006) 015.

[64] E. A. Ivanov and V. I. Ogievetsky, Teor. Mat. Fiz. 25, 164 (1975).

[65] I. McArthur, J. High Energy Phys. 11 (2010) 140.

[66] A. Anabalon, J. Gomis, K. Kamimura, and J. Zanelli, J. High Energy Phys. 10 (2006) 068. 